\title{
Metformin interferes with bile acid homeostasis through AMPK-FXR crosstalk
}

\author{
Fleur Lien, 1,2,3,4 Alexandre Berthier, ${ }^{1,2,3,4}$ Emmanuel Bouchaert, 1,2,3,4 Céline Gheeraert, 1,2,3,4 \\ Jeremy Alexandre,1,2,3,4 Geoffrey Porez,1,2,3,4 Janne Prawitt,,1,2,3,4 Hélène Dehondt,1,2,3,4 \\ Maheul Ploton, 1,2,3,4 Sophie Colin, $1,2,3,4$ Anthony Lucas, ${ }^{1,2,3,4}$ Alexandre Patrice, 1,5 \\ François Pattou, ${ }^{1,5}$ Hélène Diemer, ${ }^{6}$ Alain Van Dorsselaer, ${ }^{6}$ Christophe Rachez, ${ }^{7}$ \\ Jelena Kamilic, ${ }^{8}$ Albert K. Groen, ${ }^{8}$ Bart Staels, ${ }^{1,2,3,4}$ and Philippe Lefebvre ${ }^{1,2,3,4}$
}

\begin{abstract}
1European Genomic Institute for Diabetes (EGID), Lille, France. ${ }^{2}$ INSERM UMR1011, Lille, France. ${ }^{3}$ University of Lille 2, Lille, France. ${ }^{4}$ Institut Pasteur de Lille, Lille, France. 5INSERM UMR859, Lille, France. ${ }^{6}$ Institut Pluridisciplinaire H. Curien, Strasbourg, France. 7Unité de Régulation Epigénétique, URA 2578 du CNRS, Département de Biologie du Développement, Institut Pasteur, Paris, France. ${ }^{8}$ Department of Pediatrics, University Medical Center Groningen, Groningen, The Netherlands.
\end{abstract}

\begin{abstract}
The nuclear bile acid receptor farnesoid $X$ receptor (FXR) is an important transcriptional regulator of bile acid, lipid, and glucose metabolism. FXR is highly expressed in the liver and intestine and controls the synthesis and enterohepatic circulation of bile acids. However, little is known about FXR-associated proteins that contribute to metabolic regulation. Here, we performed a mass spectrometry-based search for FXR-interacting proteins in human hepatoma cells and identified AMPK as a coregulator of FXR. FXR interacted with the nutrient-sensitive kinase AMPK in the cytoplasm of target cells and was phosphorylated in its hinge domain. In cultured human and murine hepatocytes and enterocytes, pharmacological activation of AMPK inhibited FXR transcriptional activity and prevented FXR coactivator recruitment to promoters of FXR-regulated genes. Furthermore, treatment with AMPK activators, including the antidiabetic biguanide metformin, inhibited FXR agonist induction of FXR target genes in mouse liver and intestine. In a mouse model of intrahepatic cholestasis, metformin treatment induced FXR phosphorylation, perturbed bile acid homeostasis, and worsened liver injury. Together, our data indicate that AMPK directly phosphorylates and regulates FXR transcriptional activity to precipitate liver injury under conditions favoring cholestasis.
\end{abstract}

\section{Introduction}

In addition to their role as lipid emulsifiers, bile acids (BAs) are now also recognized as important regulators of cholesterol, bile acid, and triglyceride and glucose metabolism, as well as of a number of signaling pathways. These diverse effects are mediated through the interaction of BAs with membrane or intracellular proteins (1), including the bile acid receptor/farnesoid X receptor (BAR/FXR). FXR is activated by primary and secondary BAs and regulates BA, lipid, and glucose homeostasis. In addition to its expression in the liver, in which it regulates BA, fatty acid, lipoprotein and glucose metabolism, FXR is also present in several other tissues, such as the intestine, in which it controls BA reabsorption, thus acting as a key regulator of the BA enterohepatic cycle (2).

Through general mechanisms shared with other nuclear receptors (NRs), FXR transcriptional activity is regulated via ligand-regulated, dynamic interactions with NR coregulators, which can exert either coactivating or corepressing activities. In vitro studies demonstrated a role for PPAR $\gamma$-coactivator $\alpha$ (PGC- $1 \alpha)$ and the arginine-methyl transferases PRMT1 and CARM1 as well as DRIP205/Med1 in coactivating FXR (3-6). Furthermore, FXR activity is sensitive to extracellular signals via posttranslational modifications, which alter its ability to transactivate target genes (7).

As a BA sensor, FXR regulates hepatic BA biosynthesis, transport, and secretion and ileal reabsorption. BA homeostasis is thus believed to rely mostly on the ileal FXR-FGF19/15-FGFR4-SHP

Authorship note: Fleur Lien and Alexandre Berthier contributed equally to this work. Bart Staels and Philippe Lefebvre are co-senior authors.

Conflict of interest: The authors have declared that no conflict of interest exists. Citation for this article: J Clin Invest. 2014;124(3):1037-1051. doi:10.1172/JCI68815 axis (8). Being also a regulator of hepatic canalicular and basolateral BA transporters, FXR plays an important role in reducing liver injury upon hepatic BA overload. In most preclinical models of intra- or extrahepatic cholestasis, FXR activation induces a favorable adaptive response to BA accumulation (9-11).

Organs are submitted to nutrient fluxes and oscillate between a fasted state and an energy-replenished postprandial state. The adaptation of their metabolic activity to controlling energy and metabolic homeostasis involves the regulation of expression and/or activity of transcriptional coactivators, such as the NAD ${ }^{+}$-dependent deacetylase sirtuin SIRT1 and/or PGC-1 $\alpha$. A signaling network linking SIRT1, PGC-1 $\alpha$, and AMPK that allows skeletal muscle cells to adapt to a decreased energy supply has been identified $(12,13)$. Similarly, the SIRT1 and the AMPK pathways act in concert in mouse liver, in which a simultaneous decrease or increase in AMPK and SIRT1 activities occurs in the refed or fasted state respectively (14). Thus, a link between cellular energy levels and NR coregulator activity has been established, which was more recently extended to SRC-2, whose ability to regulate the basal expression level of the FXR target gene Bsep is positively controlled by AMPK (15). Thus, although not formally tested, the possibility arises that the metabolic status of cells impinges on FXR transcriptional activity through an interaction with energy-sensitive transcriptional coregulators, which is supported by the demonstration that glucose induces the expression of FXR (16).

Here we explore this possibility and show that AMPK interacts physically with, phosphorylates, and represses ligand-induced FXR transcriptional activity by decreasing its interaction with transcriptional coactivators. Several pharmacological AMPK activators, including metformin, repress FXR transcriptional activity 


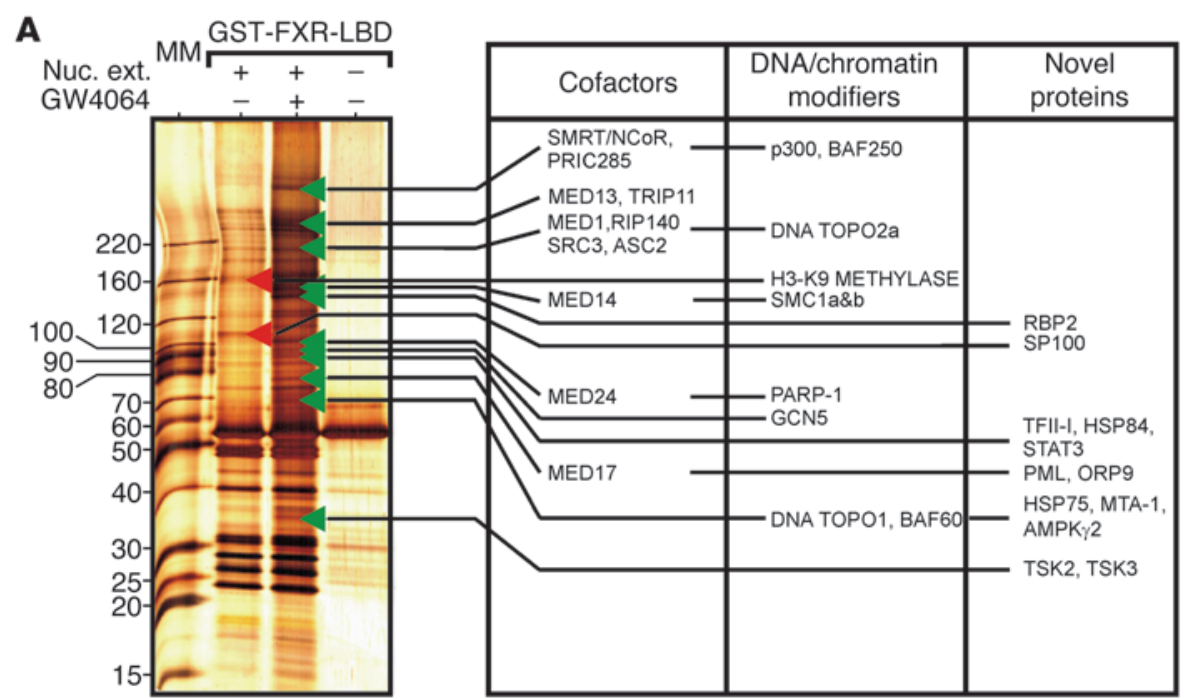

C
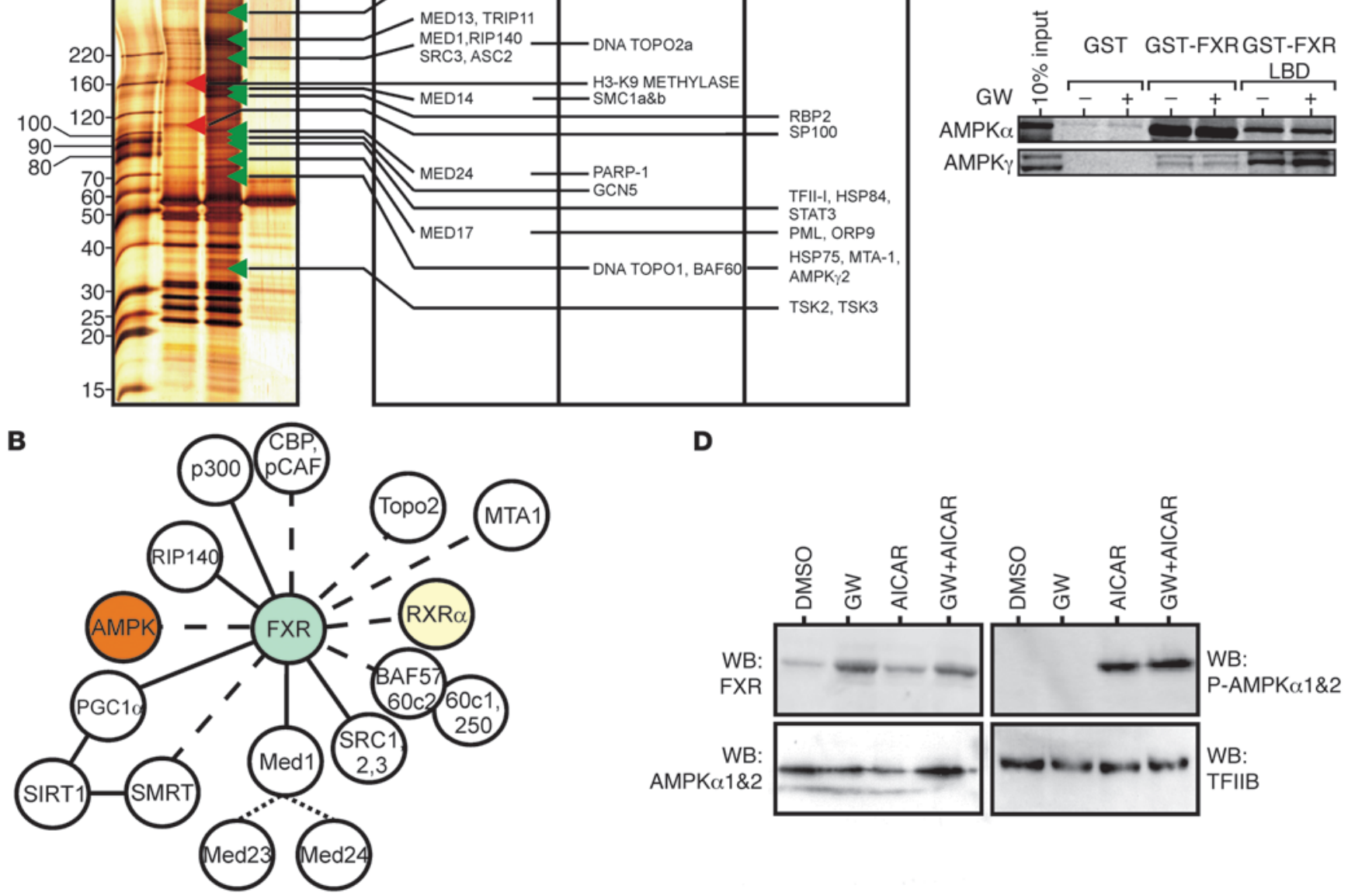

D

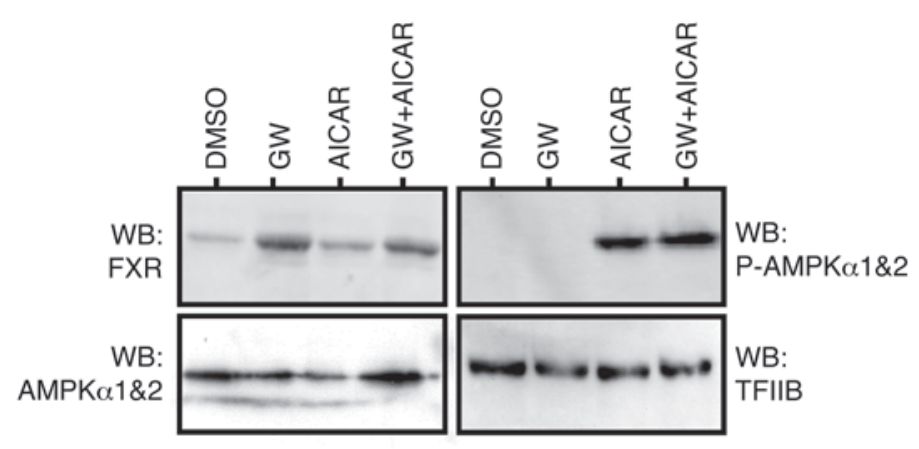

E

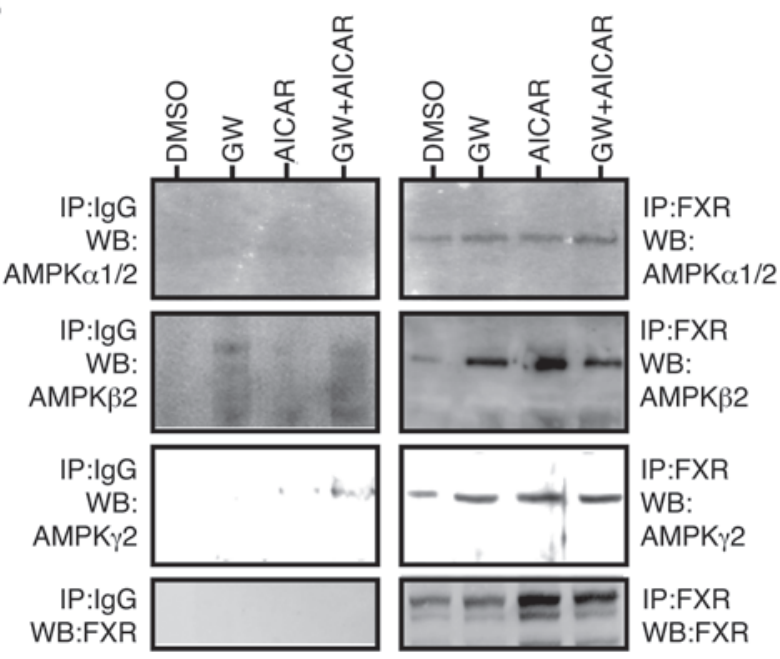

$\mathbf{F}$

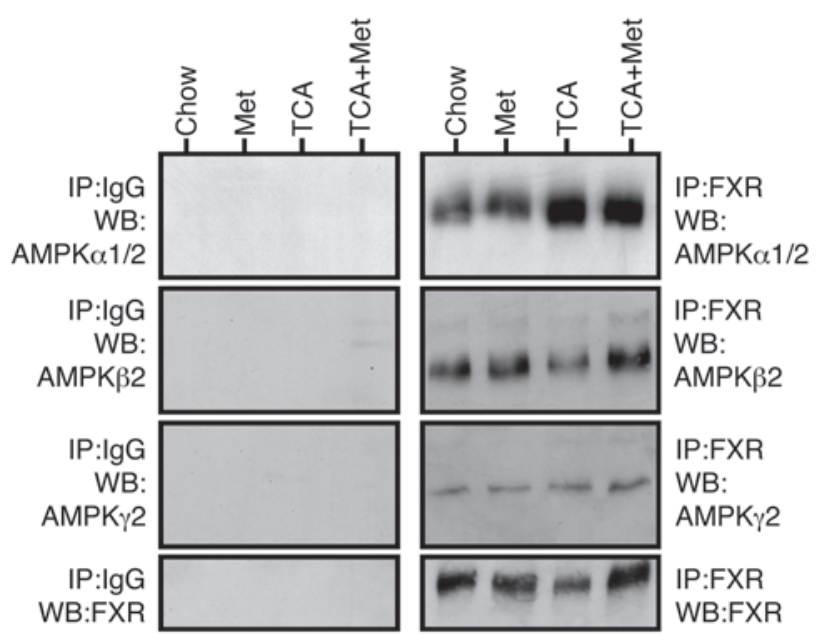




\section{Figure 1}

Identification of FXR-associated cofactors. (A) Representative silverstained acrylamide gel showing polypeptides from HepG2 extracts specifically bound to unliganded FXR LBD (-GW4064) or liganded LBD (+GW4064). Arrowheads indicate excised bands processed for mass spectrometry analysis. Selected proteins yielded a peptide coverage above $20 \%$ and were detected at least twice out of 3 analyses. (B) Detected interactions with FXR. Interactions validated by GST pulldowns are indicated here. Solid line: ligand-dependent interactions; dotted line: constitutive interactions. (C) FXR interacts with both AMPK $\alpha 1$ and AMPK $\gamma 1$. GST pull-down experiments were carried out using GSTfused fIFXR (GST-FXR) or FXR LBD (GST-FXR LBD) and ${ }^{35}$ S-labeled AMPK $\alpha 1$ and AMPK $\gamma 1$. (D and E) AMPK coimmunoprecipitates with FXR. HepG2 cells were treated with $2 \mu \mathrm{M}$ GW4064 and/or $1 \mathrm{mM}$ AICAR for 2 hours. (D) Western blot analysis of whole-cell extracts was performed to assess the presence of the indicated proteins. (E) Wholecell extracts were immunoprecipitated with either a nonspecific IgG or an anti-FXR antibody. Immunoprecipitated material was analyzed by Western blot using the indicated antibodies. Western blot analysis was carried out to assess AMPK and FXR expression levels in whole cell extracts (upper panel). (F) AMPK coimmunoprecipitates with FXR in mouse liver extracts. Mice received the indicated treatment (see Figure 6 for details), and FXR was immunoprecipitated as in $\mathbf{E}$.

in vitro and in vivo. In a model of liver injury induced by a BA overload, metformin induced FXR phosphorylation and increased plasma BA levels in a FXR-dependent manner. Furthermore, metformin also worsened liver injury in a model of chemically induced intrahepatic cholestasis. Our results thus identify AMPK as a direct regulator of BA-activated FXR.

\section{Results}

Mass spectrometry identifies components of the PRIC285 and AMPK complexes as FXR interacting proteins. Proteins from human hepatoma HepG2 cells bound to a FXR ligand-binding domain (LBD) affin- ity matrix in the presence, or not, of the synthetic FXR agonist GW4064 were resolved by SDS-PAGE. Tryptic peptides were identified by mass spectrometry (Figure 1A). Mapped peptides corresponded to Mediator complex components (Med1/DRIP205, Med13, Med14, Med24, and Med17) and PRIC complex subunits (17), such as the known coactivators or coactivator-binding proteins (PRIC285, CBP, SRC-3, PGC-1 $\alpha$ ). Other coregulators such as p300, SWI/SNF complex subunits (BAF250 and BAF60), MTA-1, and RIP140 were also identified. Finally, a more heterogenous group of polypeptides with various biological functions was identified, including the AMPK $\gamma 2$ regulatory subunit $(\mathrm{AMPK} \gamma 2)$ as well as PARP-1 and DNA topoisomerases.

mRNAs coding for these identified FXR-binding proteins were detected in HepG2, cells and their expression was not, in most cases, modified upon GW4064 treatment (Supplemental Figure 1; supplemental material available online with this article; doi:10.1172/ JCI68815DS1). Protein-protein interactions, as well as their ligand dependency, were assessed by GST pull-down assays (Supplemental Figure 2) and are summarized in Figure 1B. Med23 and Med24, components of the Mediator complex, did not interact with FXR, suggesting that our initial procedure isolated proteins binding both directly and indirectly to FXR. Previously described FXR coregulators such as $\mathrm{p} 160$ proteins, as well as Med1, PGC-1 $\alpha$, and RIP140, interacted with the full length (fl) FXR in a ligand-dependent manner. All other putative FXR coregulators, including AMPK $\gamma 2$, interacted with the FXR LBD in a ligand-insensitive manner (Figure 1, $B$ and C, and Supplemental Figure 2). Since functional links between SMRT, PGC-1 $\alpha$, SIRT1, and AMPK suggest that they constitute an integration platform for metabolic signals $(18,19)$, we evaluated whether FXR can interact physically with proteins of this platform (Supplemental Figure 2, B and C). Both flFXR and FXR LBD interacted with PGC-1 $\alpha$ (Supplemental Figure 2B). SIRT1 did not interact directly with FXR (Supplemental Figure 2B), suggest-
A

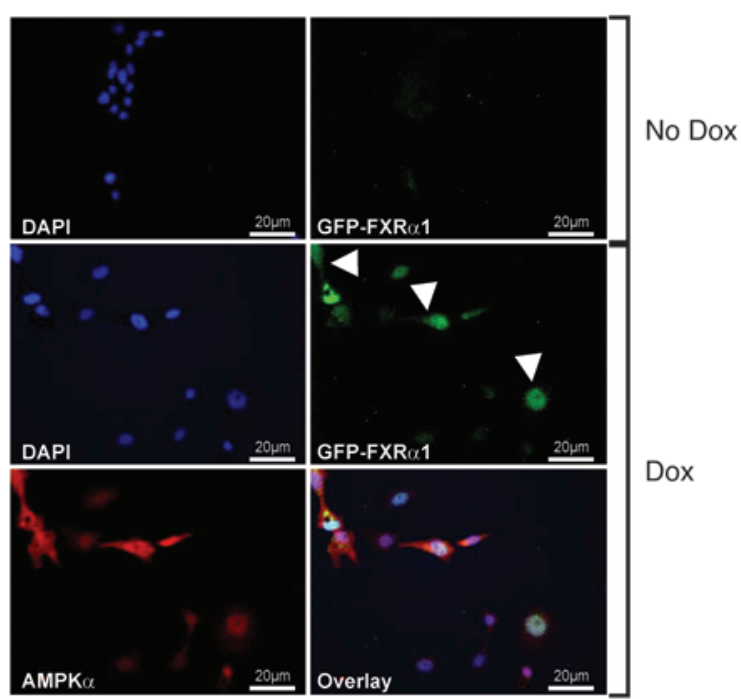

B

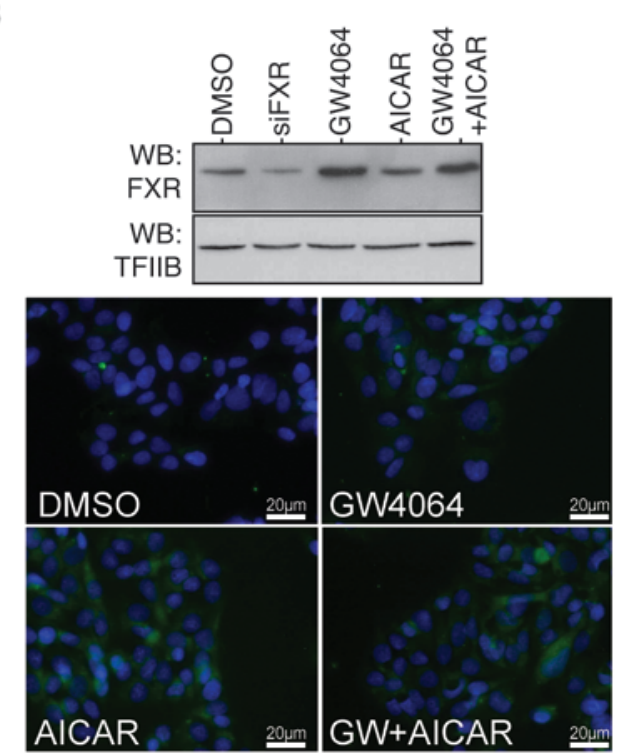

\section{Figure 2}

FXR interacts with AMPK in living cells. (A) A GFP-FXR fusion protein colocalizes with AMPK in immortalized mouse hepatocytes. The doxycyclindependent induction of GFP-FXR expression was induced or not in AML12 cells, and the localization of FXR and AMPK $\alpha$ was determined by (immuno)fluorescence. (B) FXR interacts with AMPK in the cytoplasm of living cells. Top panel: Western blot analysis of HepG2 cell extracts. Bottom panels: A PLA was performed in fixed HepG2 cells after indicated treatment with $2 \mu \mathrm{M}$ GW4064 and/or 1 mM AICAR for 2 hours. 
A

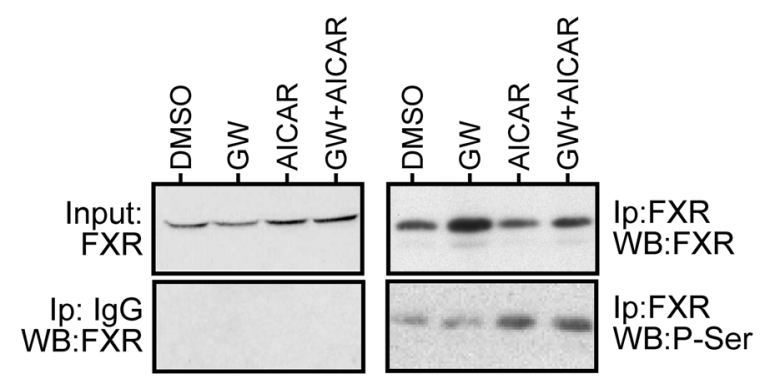

B

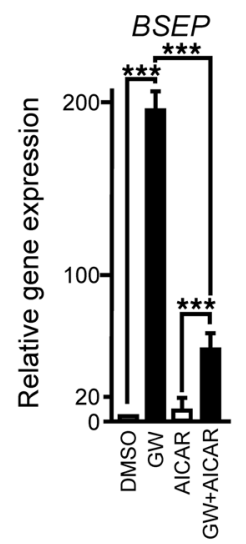

HepG2

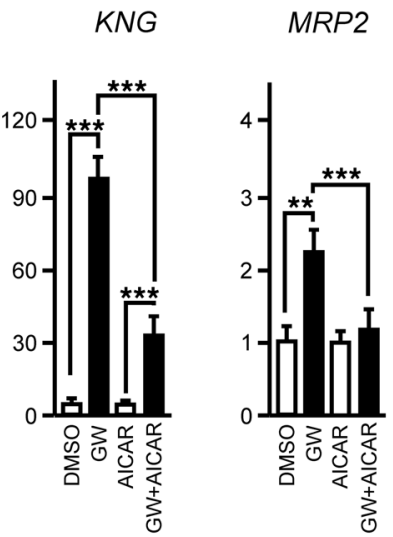

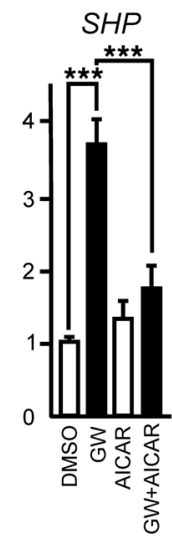

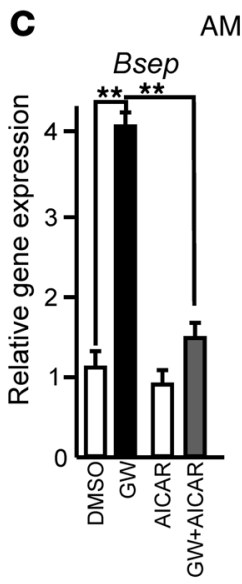

AML12
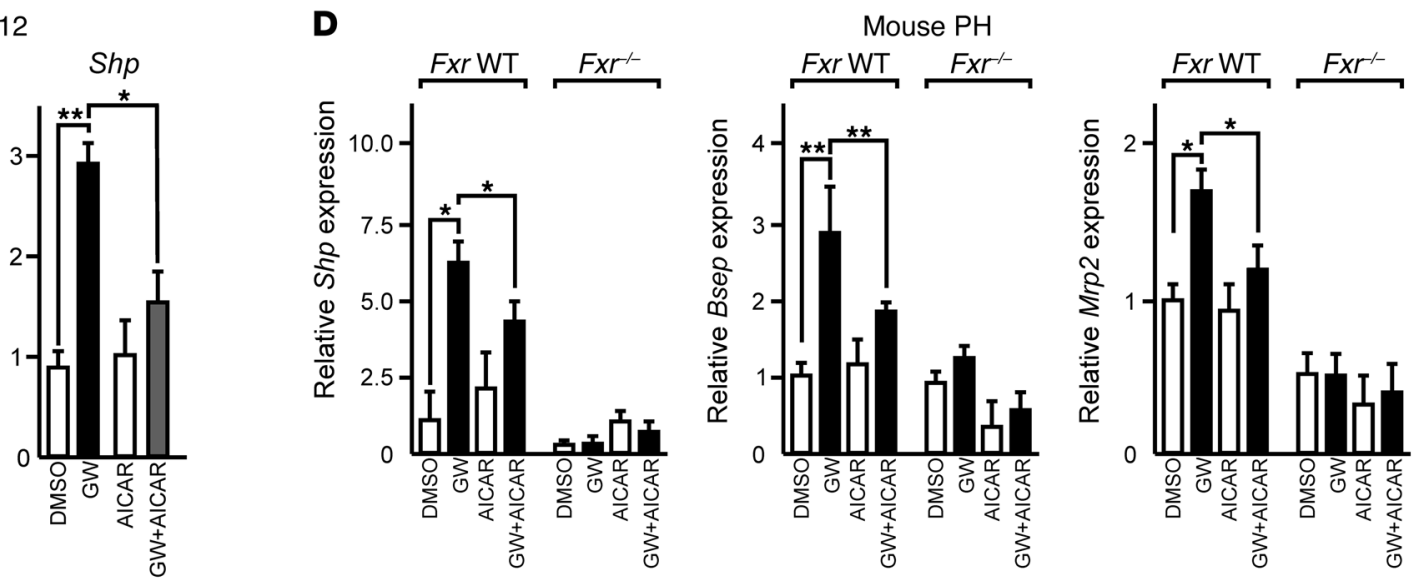

Figure 3

AMPK activation represses FXR transcriptional activity in human or mouse hepatocytes in vitro and in vivo. (A) FXR phosphorylation on Serine residues. HepG2 cells were treated with vehicle (DMSO), $2 \mu \mathrm{M}$ GW4064 (GW), or GW4064 and 1 mM AICAR (GW+AICAR). Whole-cell extracts were immunoprecipitated with an anti-FXR antibody or nonspecific IgG. Precipitates were analyzed by Western blot analysis as indicated. (B) AMPK activation represses FXR-mediated gene expression. HepG2 cells were treated as in $\mathbf{A}$, and total RNA was analyzed after 18 hours by RT-qPCR. Results are expressed relative to the mRNA level in untreated cells (set to 1). (C) Gene mRNA levels were measured in AML12 cells treated under conditions similar to those in $\mathbf{B}$, and results are expressed as in B. (D) Mouse primary hepatocytes (PH) were isolated from 12-week-old male mice and stimulated for 6 hours. RNA levels were analyzed as in $\mathbf{C}$, and results are expressed as in $\mathbf{B}$. ${ }^{*} P<0.05 ;{ }^{* \star} P<0.01 ;{ }^{* \star \star} P<0.005$.

ing that either PGC-1 $\alpha$ or SMRT bridges this deacetylase to FXR, as both interacted with SIRT1 (Supplemental Figure 2C).

While AMPK $\gamma 2$ displayed weak binding to flFXR, the catalytic $\alpha 1$ subunit of AMPK interacted strongly in this assay in a ligandindependent manner with flFXR, but more weakly with the FXR LBD (Figure 1C). The interaction of FXR with the native trimeric AMPK complex was then assessed in a coimmunoprecipitation assay prior to which HepG2 cells were treated with a combination of GW4064 and AICAR, an activator of AMPK. GW4064 stabilized FXR protein expression, and AICAR induced AMPK $\alpha$ subunit phosphorylation at T172 as expected (Figure 1D). The coimmunoprecipitation assay demonstrated a constitutive association of FXR with native AMPK (Figure 1E), corroborating the results of GST pull-down assays. Finally, liver FXR coimmunoprecipitated with AMPK subunits in mice that received either a chow diet ora diet supplemented with the BA TCA, the AMPK activator metformin, or both compounds (Figure 1F).

Both FXR and AMPK have been described as shuttling from the cytoplasmic to the nucleus compartment and vice versa $(20,21)$.
Endogenous FXR localized mostly to the nucleus of HepG2 cells, with faint staining of the cytoplasm, whereas AMPK showed a ubiquitous cellular repartition (Supplemental Figure 3). This cytoplasmic colocalization was also observed in murine immortalized AML12 cells expressing a GFP-mFXR $\alpha 1$ protein in a doxycyclindependent manner (Figure 2A). A proximity ligation assay (PLA) was thus performed in HepG2 cells, in which endogenous FXR expression increased upon agonist treatment (Figure 2B). The PLA signal from endogenous FXR and AMPK was only detected in the cytoplasm upon AICAR-mediated AMPK activation (Figure 2B), showing that a more stable AMPK-FXR interaction occurs in the cytoplasmic compartment of HepG2 cells. Together, these results demonstrate that FXR colocalizes and interacts with AMPK both in vitro and in vivo.

AMPK inbibits FXR transcriptional activity in vitro and in vivo. The physical interaction of FXR with AMPK raised the possibility that FXR could be a substrate for this kinase. HepG2 cells were thus treated as above by AICAR and/or GW4064 and the phosphorylation status of FXR was examined after immunoprecipitation 

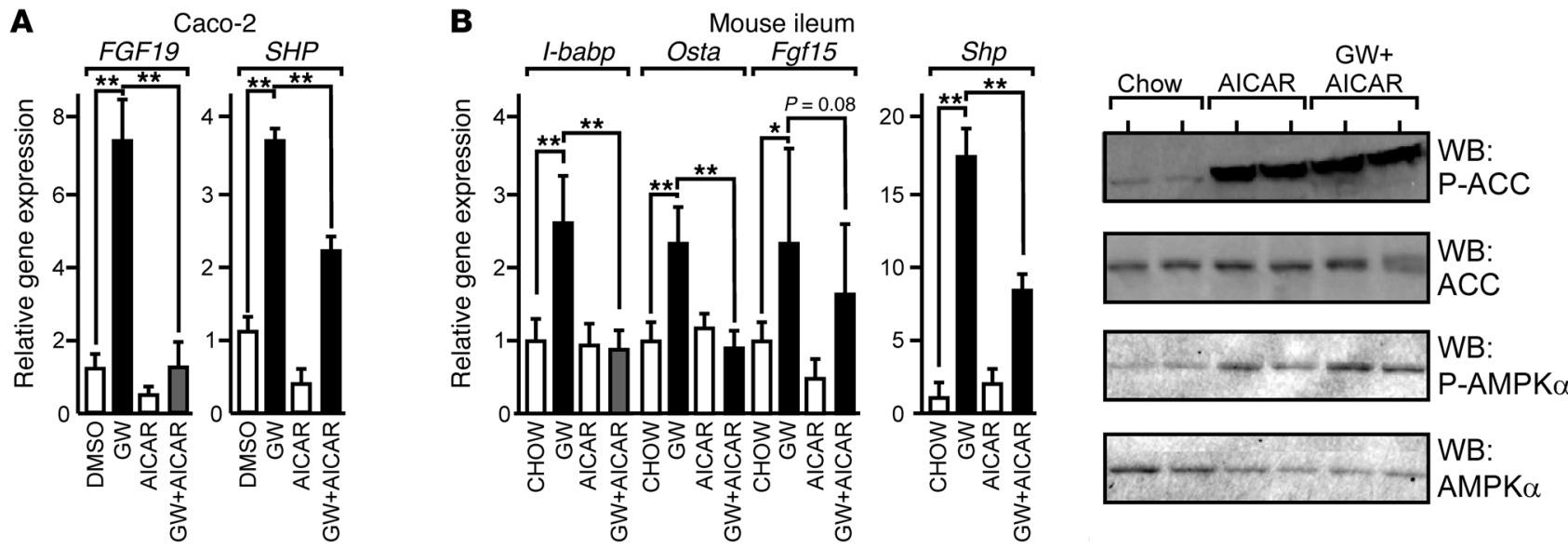

Figure 4

AMPK activation represses FXR transcriptional activity in human and mouse intestinal cells in vitro and in vivo. (A) FXR target gene expression in differentiated enterocyte-like Caco-2/TC7. Differentiated Caco-2/TC7 cells were treated with $2 \mu \mathrm{M}$ GW4064 and/or $1 \mathrm{mM}$ metformin for 2 hours. Gene expression levels were assessed by RT-qPCR assays. (B) Left panel: FXR target gene expression in C57BL/6 mice. GW4064 (30 mpk/d by gavage in $1 \%$ CMC, $1 \%$ Tween 80 ) and/or AICAR (200 mpk/d by intraperitoneal injection) were administered to mice $(n=6-8)$ for 5 days. Basal gene expression was arbitrarily set to 1 for a randomly selected mouse fed a chow diet (control), and all results are expressed relative to this basal level. Right panel: AMPK activation in AICAR-treated mice. Whole-cell extracts from mouse ilea were analyzed by Western blotting using the indicated antibodies. ${ }^{*} P<0.05 ;{ }^{* *} P<0.01$.

to show a marked increase in phosphoserine content upon concomitant activation of AMPK and of FXR (Figure 3A). Expression of endogenous FXR target genes in response to AMPK activation was then assessed in hepatoma HepG2 cells (Figure 3B), immortalized mouse hepatocytes AML12 (Figure 3C), or primary mouse hepatocytes (Figure 3D). Since variable induction kinetics were observed for each endogenous target gene (Supplemental Figure 4, $\mathrm{A}$ and $\mathrm{B}$ ), induction times were adjusted for each target gene to achieve maximal induction by GW4064. Activation of AMPK with AICAR resulted in a potent repression of GW4064-induced increase in BSEP, kininogen (KNG), MRP2, and SHP mRNAs in HepG2 cells (Figure 3B) as well as other known direct FXR target genes (Supplemental Figure 4C). A similar result was obtained in AML12 (Figure 3C) and primary mouse hepatocytes, in which this repression was shown to be FXR dependent (Figure 3D). As SHP and $K N G$ genomic loci possess distinct chromatin architectures in HepG2 cells, as shown by H3K4me3 ChIP-Seq profiles (Supplemental Figure 5), it is unlikely that the AMPK effect stems from an action through specific structural components of their promoters.

Thus, activation of the energy-sensing kinase AMPK significantly affects FXR-mediated transcription in human $(P<0.05)$ and mouse (for Bsep, $P<0.001$; for Shp and Mrp2, $P<0.05$ ) hepatic cells. This effect was equally observed in human enterocyte-like Caco-2/TC7 cells (Figure 4A) and further assessed in vivo. WT C57BL/6 mice, which display normal plasma glucose levels, were gavaged with GW4064 (30 mpk/d) and injected intraperitoneally with $\operatorname{AICAR}(200 \mathrm{mpk} / \mathrm{d}$ ) for 5 days (Figure 4B). Importantly, these treatments did not alter blood glucose and lipid levels (Supplemental Figure 6). As the limited bioavailability of GW4064 did not allow for monitoring FXR target gene activation in the liver, we assessed the expression of FXR target genes in ileum, in which AICAR activated AMPK and led to the phosphorylation of its substrate ACC in ileum (Figure 4B). Under these conditions, the FXR target genes Osta, Shp, Fgf15, and I-babp/Fabp6 were significantly induced upon GW4064 treatment (for Osta, Shp, and
I-babp/Fabp6, P < 0.01; for Fgf15, $P<0.05$ ). While AICAR administration had by itself no effect on the basal expression level of these genes, it significantly blunted the GW4064-induced expression of Osta, Shp, and I-babp/Fabp6 $(P<0.01)$. Fgf15 expression exhibited a similar, albeit nonsignificant, trend. Taken together, our data suggest that AMPK activation blunts FXR transcriptional activity both in vitro and in vivo.

FXR is a substrate for AMPK-catalyzed phosphorylation. The physical interaction of FXR with native AMPK prompted us to determine whether FXR could be a substrate for this kinase. An in vitro phosphorylation assay using purified $\alpha 2 / \beta 2 / \gamma 1$ AMPK subunits (wtAMPK 2:2:1) together with full-length $\mathrm{His}_{6}$-tagged mFXR $\alpha 3$ revealed that FXR is phosphorylated by wtAMPK and, more efficiently, by a constitutively active AMPK complex (ref. 22; CA AMPK [2:2:1]) (asterisks, Figure 5A). Similar results were obtained with the trimeric AMPK 1:1:2 complex (data not shown). We then identified the phosphorylated residue(s) by tandem mass spectrometry using purified mFXR $\alpha 3$ as a substrate, for which a bioinformatic prediction (Scansite) identified a single potential phosphoserine residue at position 250. LC-MS/MS analysis was conducted in parallel on chymotrypsic digests of purified, native mFXR $\alpha 3$ (FXR $\alpha 3$ control) and mFXR $\alpha 3$ after in vitro phosphorylation with AMPK and allowed a sequence coverage of $97 \%$. MS/MS data interpretation of the corresponding CID and ETD MS/MS spectra unambiguously identified Ser250 in 2 chymotrypsin digests of the mFXR $\alpha 3$ phosphorylated sample (Figure $5 \mathrm{~B}$ ), which was confirmed by additional analysis of pepsin and AspN digests. No molecular ion corresponding to a phosphorylated form of any peptide could be observed in the FXRa3, nonphosphorylated control sample. FXR S250 is conserved in the human and mouse FXR isoforms (Figure 5C). An antibody raised against a phospho-S250-containing peptide (RDLRQVT[PS]TTKFC) recognized specifically the AMPKphosphorylated form of FXR (Figure 5D). To test whether AMPK activation could affect FXR intrinsic transcriptional activity, a 


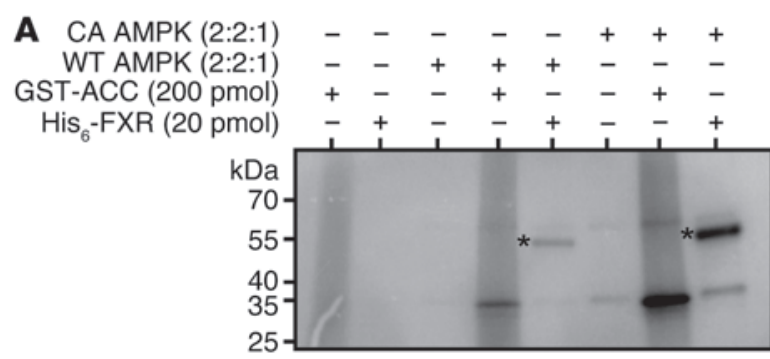

B
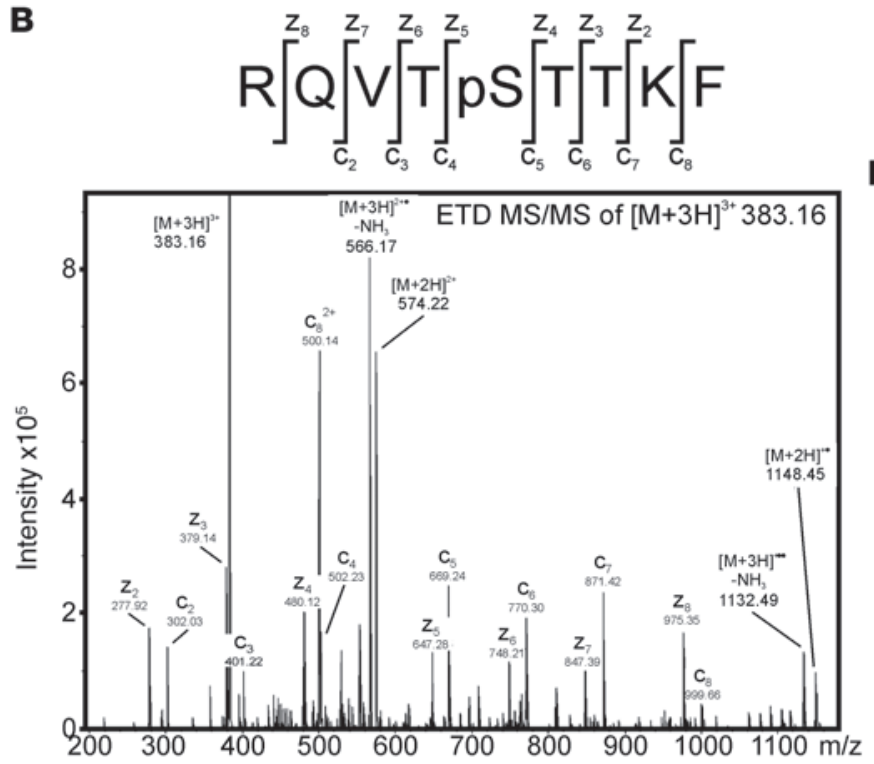

C Mouse FXRa3

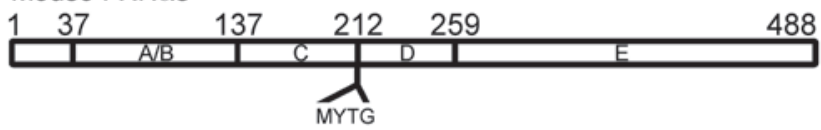

Mouse FXR 1 226-EGRDLRQVTS TTKFCREKTE LTADQQ Mouse FXR 22 222-EGRDLRQVTS TTKFCREKTE ITADQQ Mouse FXR $\alpha 3$ 240-EGRDLRQVTS TTKFCREKTE LTADQQ Mouse FXR $\alpha$ 236-EGRDLRQVTS TTKFCREKTE ITADQQ

Human FXR $\alpha 1$ 228-EGRDIRQVTS TTKSCREKTE ITADQQ Human FXR $\alpha 2$ 224-EGRDLRQVTS TTKSCREKTE ITADQQ Human FXR $\alpha 3$ 238-EGRDLRQVTS TTKSCREKTE LTADQQ Human FXR $\alpha 4$ 234-EGRDLRQVTS TTKSCREKTE LTADQQ

D

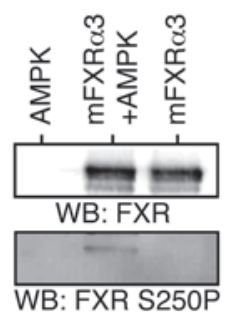

E

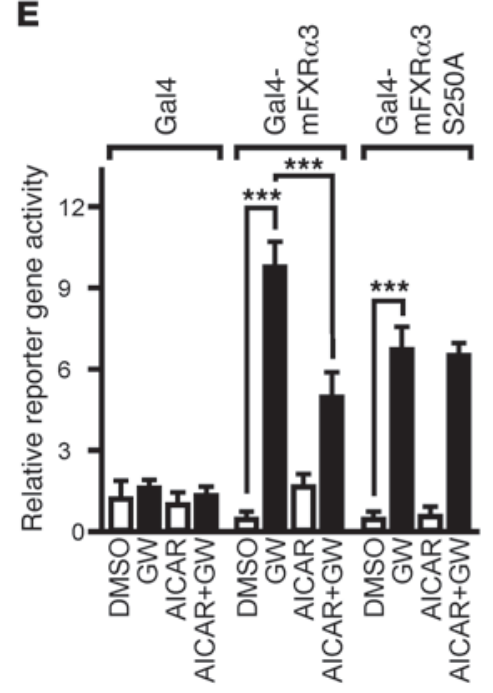

Figure 5

AMPK phosphorylates FXR on a single serine residue. (A) Mouse FXR is phosphorylated by AMPK in vitro. Purified GST-ACC (positive control), $\mathrm{His}_{6}$-tagged $\mathrm{mFXR} \alpha 3$ were incubated together with purified wtAMPK or constitutively active (CA) AMPK and ${ }^{32}$ P-ATP and resolved by SDS-PAGE. (B) The ETD MS/MS spectrum of chymotrypsin peptide 246-254 (RQVTSTTKF, 383.163+) from phosphorylated mFXR 23 showing phosphorylation at Ser250. (C) Sequence conservation of the hinge region of mouse and human FXR isoforms. (D) An anti-phospho-S250 peptide recognizes

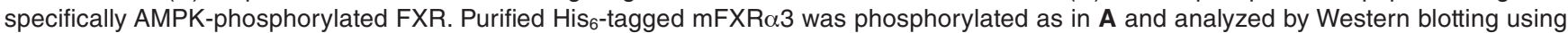
anti-FXR (FXR) or the anti-S250P FXR antibody. (E) The S250A mutation protects FXR from AICAR-induced inactivation. HepG2 cells were transfected with a Gal4-responsive luciferase reporter gene and an expression vector coding for a Gal4 DBD-wtFXR (mFXR $\alpha 3$ ) fusion protein or its mutated counterpart. ${ }^{\star \star \star} P<0.005$.

1-hybrid assay was used in which the fl mFXR $\alpha 3$ was expressed as a Gal4 DNA-binding domain fusion protein in HepG2 cells (Gal4-mFXRa3, Figure 5E). AICAR strongly inhibited agonistinduced $\mathrm{mFXR} \alpha 3$ transcriptional activity, and a similar result was also observed for the murine Gal4-mFXR $\alpha 4$ expressed in AML12 cells (Supplemental Figure 7) and human $\alpha 2$ isoform in HepG2 cells (Figure 6F). Preventing FXR phosphorylation by introducing an S-to-A mutation at position $250 \mathrm{in} \mathrm{mFXR} \alpha 3$ abrogated the inhibitory effect of AICAR, although we noted that this mutation also partially decreased the ligand responsiveness of FXR (Figure 5E). Of note, systematic mutagenesis of all other potential AMPK phosphorylation sites (S62, S65, S117, T156, S238), as determined by a low stringency bioinformatic scanning of the human FXR $\alpha 2$ sequence, did not confer resistance to AICAR treatment (Supplemental Figure 8). Taken together, we demonstrate here that AMPK phosphorylated FXR on a single amino acid, hence blunting the transcriptional activity in response to agonist stimulation.
AICAR and metformin inbibit FXR transcriptional activity in an AMPKdependent manner. As the purified human FXR $\alpha 2$ and $\alpha 3$ isoforms (GST-hFXR $\alpha 2$ and $\mathrm{His}_{6}-\mathrm{h} F X R \alpha 3$ ) are phosphorylated by AMPK (Figure 6A), we aimed at determining whether the AMPK pathway crosstalks with FXR on isolated FXR response elements (FXRE). First, siRNA silencing experiments were performed to investigate whether coregulators potentially sensitive to AMPK modulate FXR transcriptional activity in HepG2 cells (Figure 6B). Efficient knockdown of SMRT or SIRT1 (Supplemental Figure 9, A and B) did not affect $K N G, B S E P$, or $F G F 19$ gene expression in response to the agonist GW4064 (Figure 6B), an observation which, combined with the lack of activity of TSA in this system (data not shown), rules out a participation of the corepressor/HDAC complex to FXR-mediated transcription in this model. Similarly, SIRT1 knockdown did not affect $K N G, B S E P$, or FGF19 gene induction, whereas PGC- $1 \alpha$ contributed marginally to FXR-controlled KNG induction. By contrast, SHP expression was strongly affected by SIRT1 and PGC-1 $\alpha$ knockdowns. Importantly, the simultaneous 

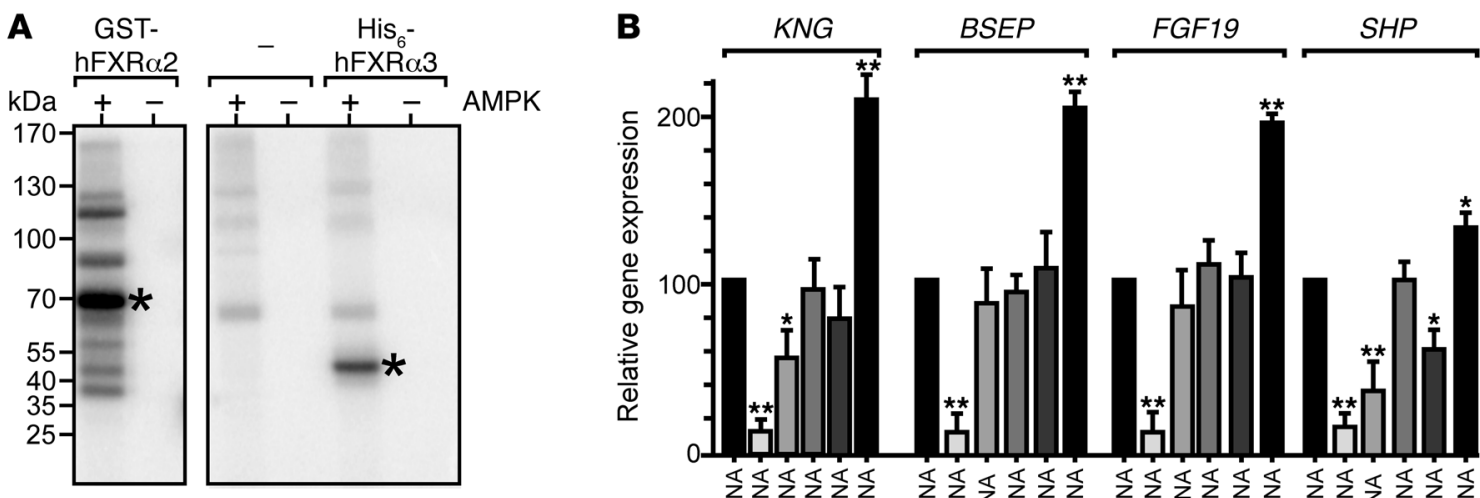

C
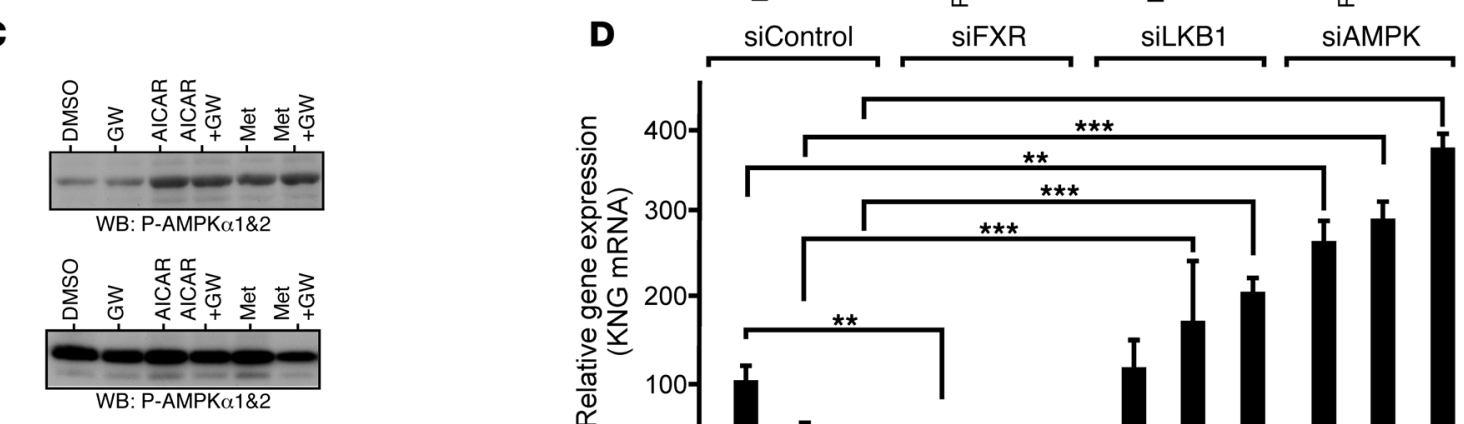

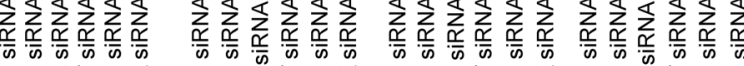

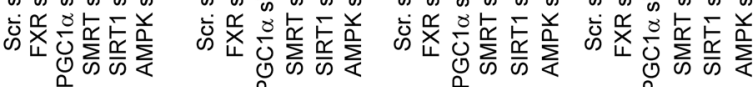
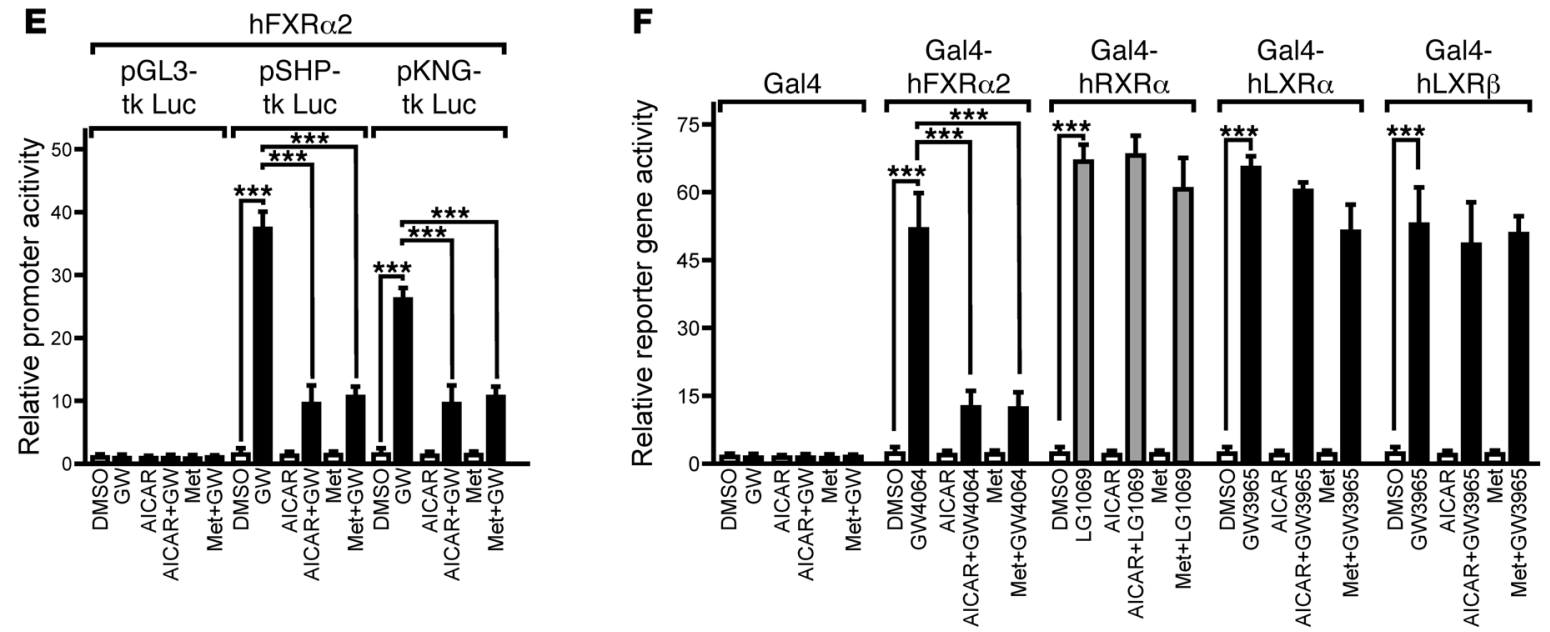

Figure 6

FXR activity is specifically inhibited by the LKB1/AMPK pathway. (A) hFXR is phosphorylated by AMPK in vitro. Partially purified GST-hFXR 2 or $\mathrm{His}_{6}$-tagged hFXR $\alpha 3$ and wtAMPK were incubated with ${ }^{32}$ P-ATP. (B) KNG, BSEP, FGF19, and SHP mRNA levels in FXR-, PGC1 $\alpha$-, SMRT-, SIRT1, or AMPK-depleted HepG2 cells. mRNA levels in siRNA-transfected cells treated with $2 \mu \mathrm{M}$ GW4064 are expressed relative to control levels in siRNA-treated cells (set at 100\%). (C) AMPK activation in HepG2 cells. Whole-cell extracts from AICAR and/or GW4064-treated cells were analyzed by Western blot using the indicated antibodies. (D) Repression of FXR activity requires a functional AMPK pathway. KNG mRNA levels were monitored in siRNA-transfected HepG2 cells. Results are expressed relative to the basal level (DMSO) set at 1. (E) AMPK activators inhibits FXRE-bound RXR/FXR heterodimers. HepG2 cells were transfected with either the reference pGL3-tk Luc reporter vector, or the same vector driven by the human SHP (pSHP-tk Luc) or KNG (pKNG-tk Luc) FXREs and expression plasmids encoding human flhRXR $\alpha$ and fIFXR $\alpha 2$. Values represent the fold activation over the activity of pGL3-tk Luc alone set to 1. (F) AMPK activators block Gal4-FXR activity. HepG2 cells were transfected with the Gal4-tk Luc reporter gene and Gal4-DBD, or Gal4-DBD fused to human flFXR 2 2 (Gal4-FXR), fIRXR $(\mathrm{Gal} 4-\mathrm{RXR} \alpha)$, or fILXR $\alpha(G a \mid 4-L X R \alpha)$ and fILXR $\beta(G a l 4-L X R \beta)$ Gal4 expression vectors. Cells were treated for 24 hours after transfection, and results are expressed as in $\mathbf{E} .{ }^{*} P<0.05 ;{ }^{* *} P<0.01 ;{ }^{* *} P<0.005$. 

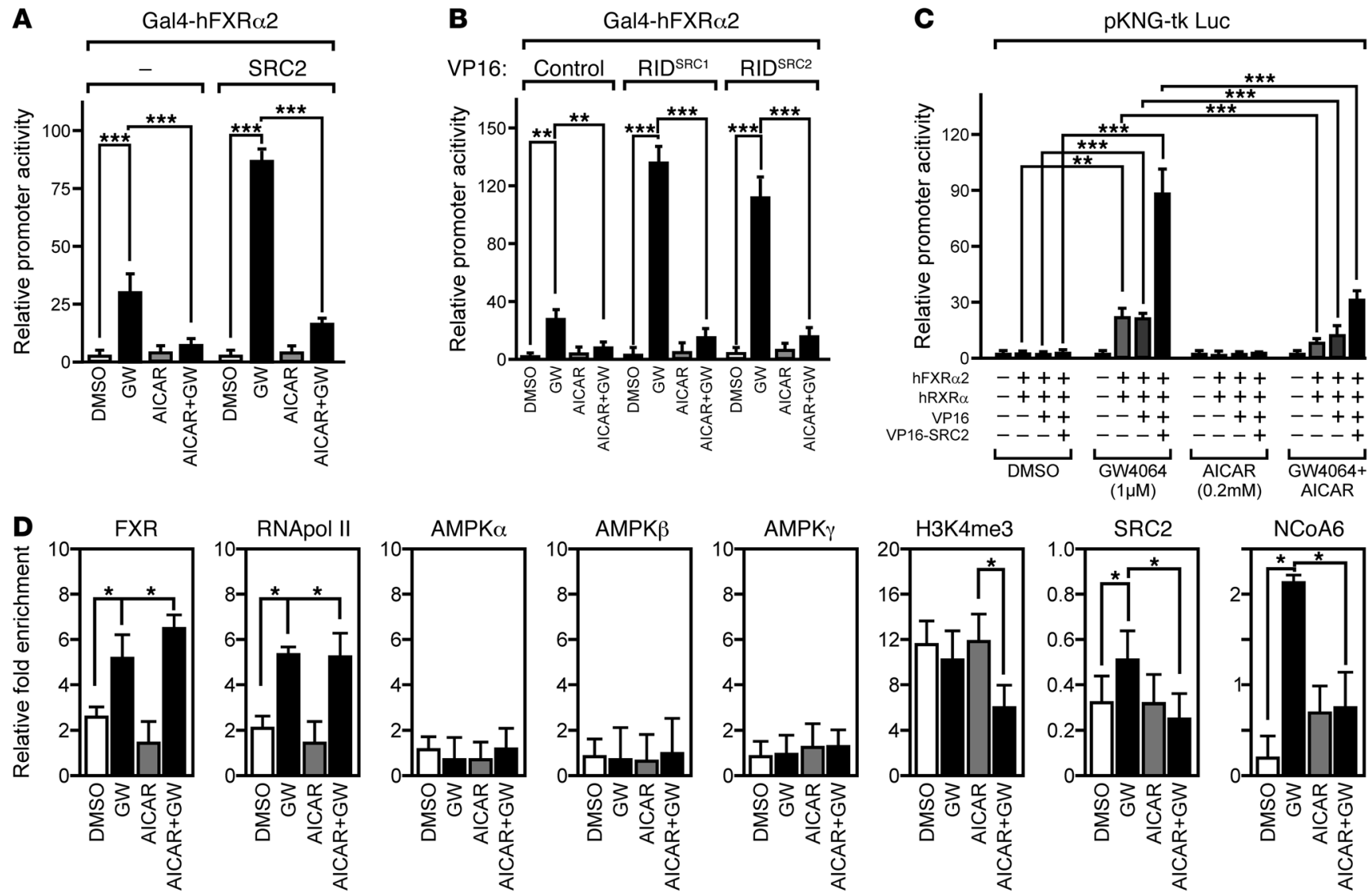

E

VP16-RID SRC2
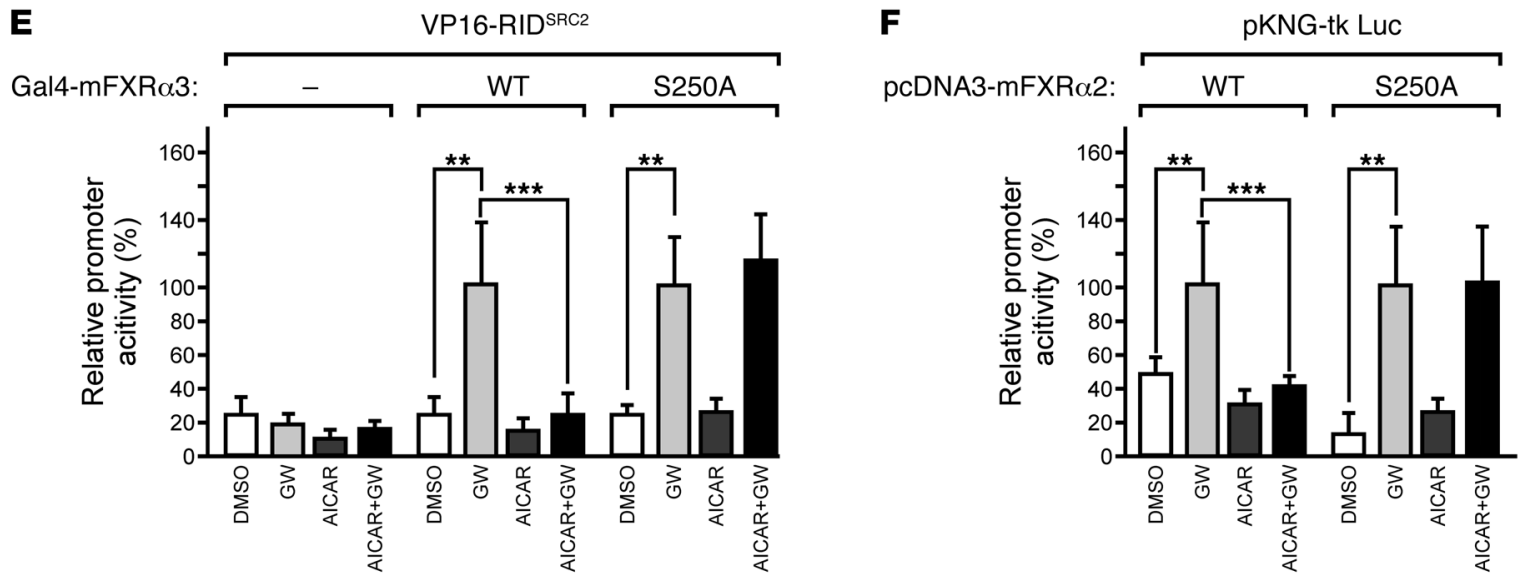

Figure 7

AMPK blocks FXR transcriptional activity through inhibition of coactivator loading. (A and B) AMPK activation prevents coactivator recruitment to FXR in a mammalian 2-hybrid assay. HepG2 cells were transfected with the Gal4-tk Luc reporter gene and a Gal4-DBD fused to human fIFXR 22 (Gal4-FXR) without (-) or with (+) an expression vector coding for fISRC-2 (A) or SRC2 RID (B) and treated for 24 hours after transfection as indicated, after which luciferase activities were assayed. Results are expressed as above. (C) AMPK activation prevents coactivator recruitment to DNA-bound FXR/RXR heterodimers. HepG2 were transfected with the pKNG-tk Luc and expression vectors coding for human fIRXR $\alpha$ and $\mathrm{hFXR} \alpha 2$, VP16 activating domain (VP16-AD), or VP16 coupled to SRC2-RID (VP16-SRC2). Cells were treated and results expressed as in A. (D) HepG2 cells were incubated with or without GW4064/AICAR for 90 minutes, crosslinked chromatin was immunoprecipitated with the indicated antibodies, and the precipitated genomic DNA purified. The KNG promoter fragment, including its FXRE, was amplified by PCR. Results were normalized to myoglobin as a negative control and are plotted as the fold enrichment over background $(n=2-3)$. (E) Mouse FXR $\alpha 3$ S250 mediates the inhibitory effect of AMPK activation on coactivator recruitment. The 2-hybrid assay was carried out as in B using Gal4-mFXRo3 or Gal4-mFXR $\alpha 3$ S250A and VP16-SRC2 RID expression vectors. (F) The mouse FXR 22 isoform is sensitive to AMPK-mediated inhibition. Transactivation assays were carried out as in $\mathbf{C} .{ }^{*} P<0.05 ;{ }^{* *} P<0.01 ;{ }^{* *} P<0.005$. 

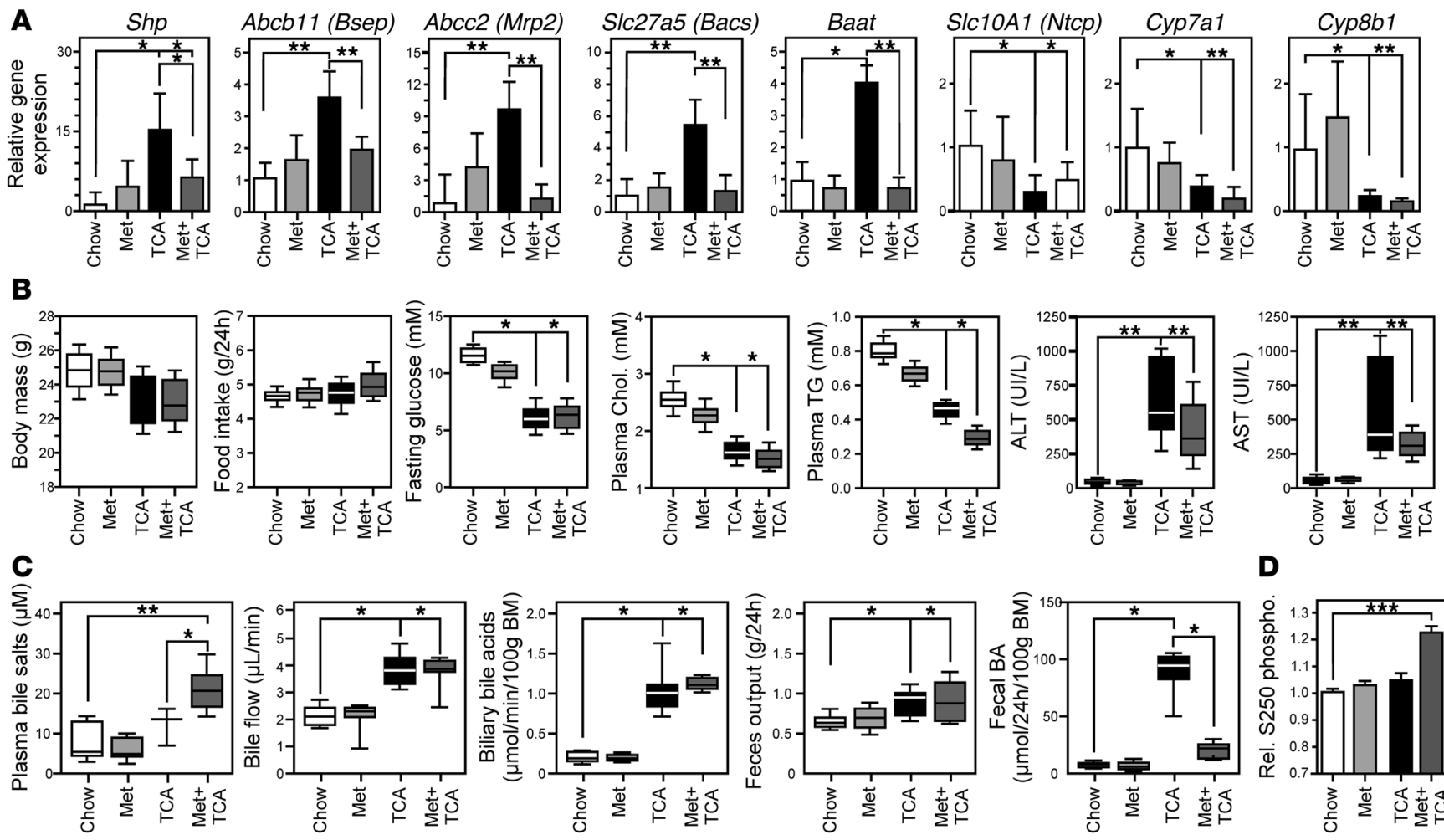

D

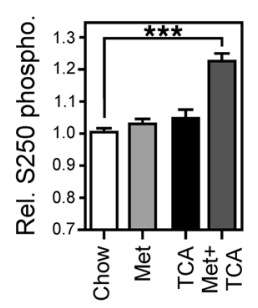

Figure 8

Metformin inhibits FXR target gene induction by TCA and BA excretion in vivo. (A) FXR target gene expression in liver. C57BL/6 mice were treated with $0.5 \%$ TCA and/or metformin (ca. 50-80 mpk/d). FXR target gene expression was monitored by RT-qPCR, and results are expressed relative to control samples (chow diet) arbitrarily set to 1. (B) Biometric and plasma biochemical parameters. (C) BA content in plasma, gallbladder, and feces. BA content was assayed as described in Methods. Results are expressed as in C. (D) Phosphorylation state of FXR in mouse livers. Bar graph represents the densitometric quantification of Western blot analysis (see Supplemental Figure 16), which is expressed as values of the phospho signal normalized to that of total FXR, relative to the chow diet signal. which was arbitrarily set to $1 .{ }^{\star} P<0.05 ;{ }^{* \star} P<0.01 ;{ }^{* * \star} P<0.005$.

knockdown of the AMPK $\alpha 1$ and $\alpha 2$ catalytic subunits (Supplemental Figure 9) revealed that AMPK represses agonist-induced $K N G, B S E P$, and FGF19 expression, whereas AMPK knockdown modulated SHP mRNA levels much less, although to a degree that was statistically significant (Figure 6B). Thus, endogenous AMPK is, in HepG2 cells, a regulator of KNG, BSEP, and FGF19 gene expression, whereas SHP displays a mixed dependency on SIRT1, PGC-1 $\alpha$, and AMPK expression levels. To ensure that the effect of AICAR or the indirect AMPK activator metformin is dependent on the upstream kinase LKB1 and/or AMPK, HepG2 cells were transfected with appropriate siRNAs and treated with these compounds (which efficiently activated AMPK, Figure 6C) and GW4064. siRNA-mediated depletion of LKB1 prevented metformin- or AICAR-induced repression of $K N G$ expression (Figure $6 \mathrm{D}$ ), demonstrating its essential role in AMPK activation in the HepG2 cell model. AMPK $\alpha 1$ and AMPK $\alpha 2$ knockdown again increased GW4064-induced KNG expression and also prevented AICAR and metformin from repressing $K N G$ expression. Therefore, AMPK is a negative regulator of multiple FXR target genes in vitro.

HepG2 cells were transfected with expression vectors coding for hFXR $\alpha 2$ and $h R X R \alpha$ and reporter genes driven by FXREs from the SHP or KNG gene promoters. AICAR and metformin at concentrations activating AMPK (Figure 6C), blunted the ability of GW4064 to induce FXR transcriptional activity in this chimeric system (Figure 6E). Thus, the use of FXR-responsive heterologous report- er systems indicated that AMPK affects the transcriptional activity of FXRE-bound, agonist-activated FXR. AMPK activation affected human FXR intrinsic transcriptional activity in a 1-hybrid assay in which the human flFXR $\alpha 2$ was expressed as a Gal4 DNA-binding domain fusion protein (Gal4-hFXR $\alpha 2$, Figure 6F). Of note, human RXR $\alpha, L X R \alpha$, and LXR $\beta$ transcriptional activities were insensitive to AMPK stimulation. In similar conditions, the activity of different FXR transcriptional modulators (NCoA1/SRC1, NCoA2/ SRC2, NCoR2/SMRT, PGC-1 $\alpha$ ) was not affected (Supplemental Figure 10A). Thus AMPK represses FXR-RXR heterodimer activity solely through an action on FXR.

AMPK activation regulates coactivator recruitment to FXR-regulated promoters. The molecular basis of the AMPK-mediated repression of FXR transcriptional activity was examined in a 2-hybrid system in which the interaction of Gal4-flhFXR $\alpha 2$ with SRC2, SRC1, or SRC3 was tested in HepG2 cells (Figure 7A and Supplemental Figure 10B, respectively). SRC2, as well as SRC1 and SRC3, albeit to a lesser extent, significantly increased the activity of agoniststimulated FXR $(P<0.01)$, and this property was blunted in the presence of AICAR $(P<0.01)$. A 2-hybrid assay assessing the interaction of FXR with the LXXLL motif-containing receptor interacting domain (RID) of SRC1 and SRC2 also demonstrated that AMPK activation inhibits this ligand-induced interaction (Figure 7B). In line with this observation, the ligand-dependent interaction of flSRC2 with FXRE-bound FXR/RXR heterodimers 
A

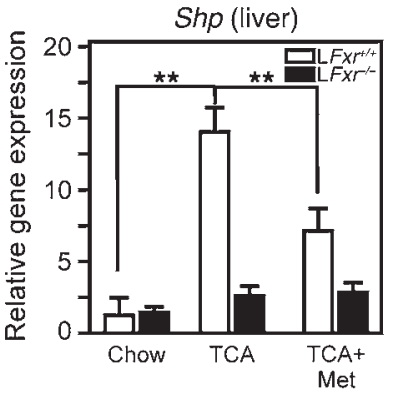

B

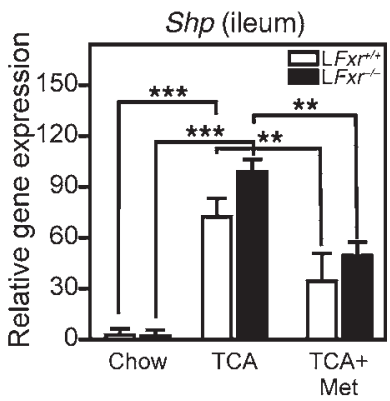

C

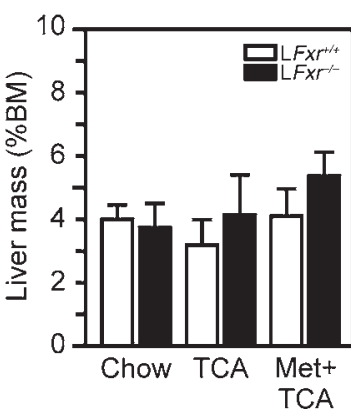

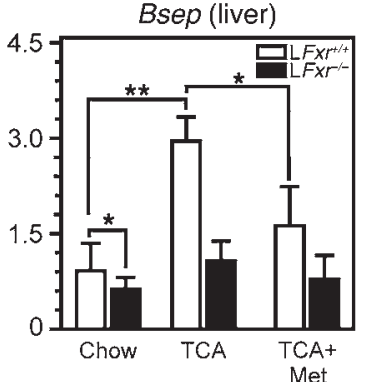
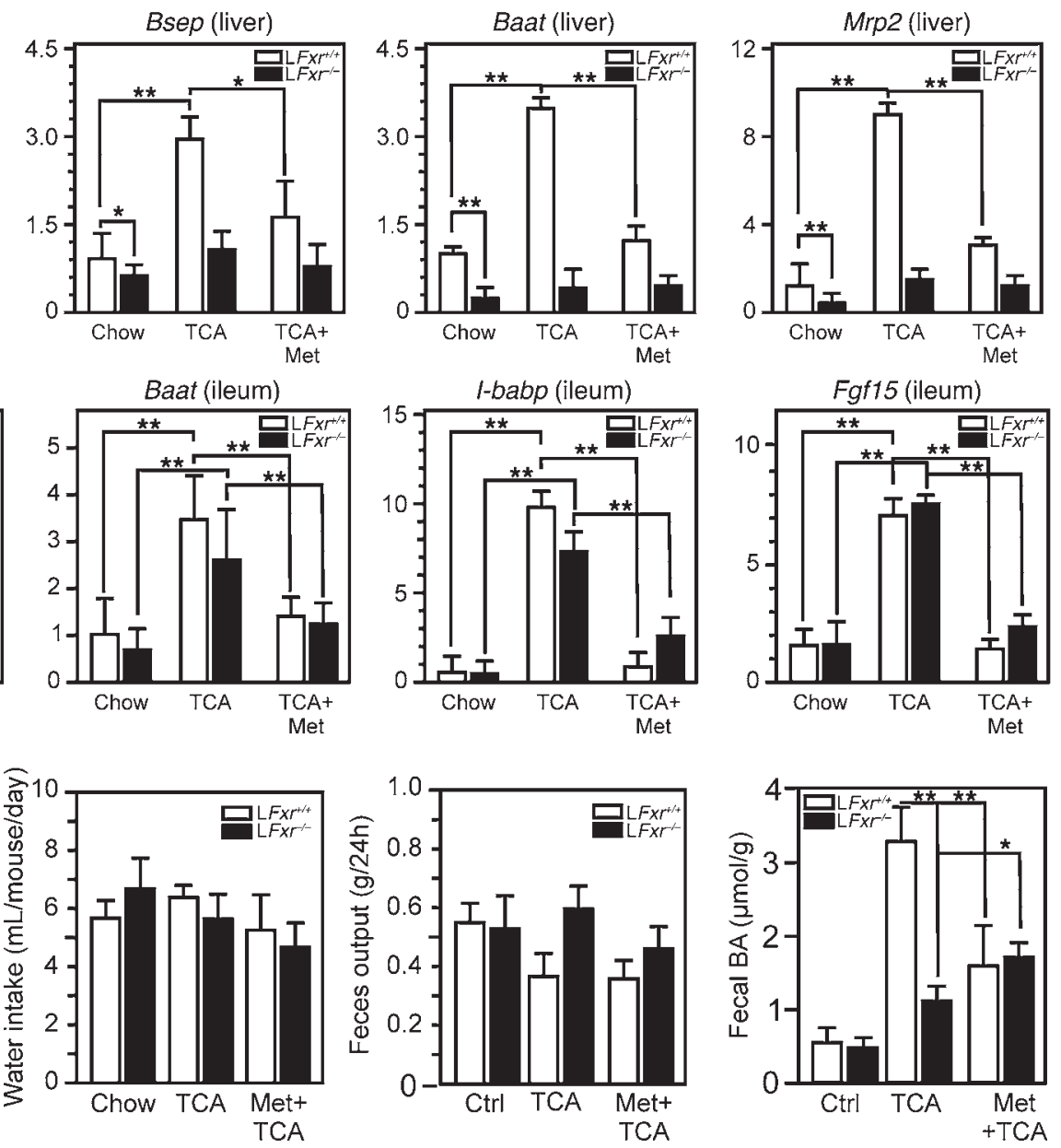

Figure 9

FXR is required to observe the inhibitory effect of metformin on FXR target genes and disrupted BA metabolism. (A) FXR target gene expression in liver of WT or FXR KO mice after TCA and/or metformin treatment. Liver FXR-KO (LFXr $\left.{ }^{-/}\right)$or WT littermates (LFXr+/+) were treated for 5 days with a chow diet supplemented (TCA) or not (chow) with 0.5\% TCA. Metformin was administered per oral administration at ca. 50-80 mpk/d. (B) lleal gene expression in WT or $\mathrm{LFx}^{-1-}$ mice after TCA and/or metformin treatment. Results are expressed as in B. (C) BA excretion in LFxr ${ }^{-1-}$ mice and WT littermates. Biometrics and biochemical assays were carried out as described in the Figure 8 legend. ${ }^{\star} P<0.05,{ }^{\star \star} P<0.01$; ${ }^{\star \star \star} P<0.005$.

was abolished upon AICAR treatment (Figure 7C). ChIP-quantitative PCR (ChIP-qPCR) assays were carried out in HepG2 cells using a ChIP-grade anti-FXR antibody (Supplemental Figure 11A). In this system, GW4064 (and CDCA; data not shown) treatment promoted the binding of FXR and RXR to the KNG FXRE (Figure 7D and Supplemental Figure 11B). Activation of AMPK in HepG2 cells by AICAR (Figure 7D), metformin, or resveratrol, another AMPK activator (Supplemental Figure 12B), or in vivo by metformin (Supplemental Figure 12) did not affect FXR loading to genomic binding sites.

Interestingly, RNA polymerase II loading to the KNG promoter was not affected by activation of AMPK by AICAR in HepG2 cells (Figure 7D). In this system, no binding of AMPK subunits was detected, in agreement with the observed cytoplasmic interaction of FXR and AMPK (Figure 2). However, AMPK activation prevented the ligand-dependent recruitment of either SRC2 or NCoA6/ASC2, which correlated with a decrease of the active promoter histone mark H3K4me3. Thus, AMPK activation prevents agonist-induced FXR interaction with transcriptional coactivators in HepG2 cells. To establish whether AMPK activation inhib- its mouse FXR, a Gal4-mFXR $\alpha 3$ fusion protein was expressed together with the SRC2-RID hooked to VP16 (Figure 7E). AICAR efficiently blunted $\mathrm{mFXR} \alpha 3$ transcriptional activity in response to agonist treatment, an effect that was abolished in the S250A mutant. In conditions inducing FXR S250 phosphorylation (Supplemental Figure 13), a similar sensitivity to AMPK activation of DNA-bound fl $\mathrm{mFXR} \alpha 2$ was observed, which was prevented by the S250A mutation (Figure 7F).

In vivo activation of AMPK by metformin blunts $B A$ induction of a subset of FXR target genes involved in BA transport and metabolism. To establish the biological role of the AMPK/FXR crosstalk, WT C57BL/6 mice were tested for their adaptive response to a BA challenge. Mice were fed either a chow diet or a chow diet supplemented with $0.5 \%$ TCA and/or metformin in drinking water to reach $50-80 \mathrm{mpk} / \mathrm{d}$, thus within the range of the clinically used dose in humans (1-2 g/d, ca $30 \mathrm{mpk} / \mathrm{d}$ ). The expression level of FXR-regulated genes involved in BA synthesis (Shp, Fgf15, Cyp7a1, Cyp8b1), BA efflux (Bsep, Mrp2, Osta/b), or uptake (Ntcp) and metabolism (Baat, Bacs) was assayed by qPCR in liver (Figure 8A). TCA treatment significantly increased $\operatorname{Shp}(P<0.05)$, Bsep, Mrp2, Baat $(P<0.01)$, 
A
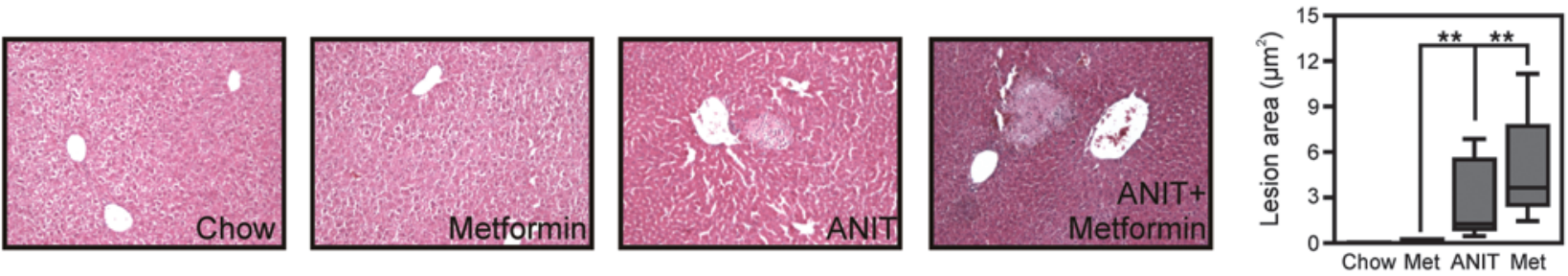

B
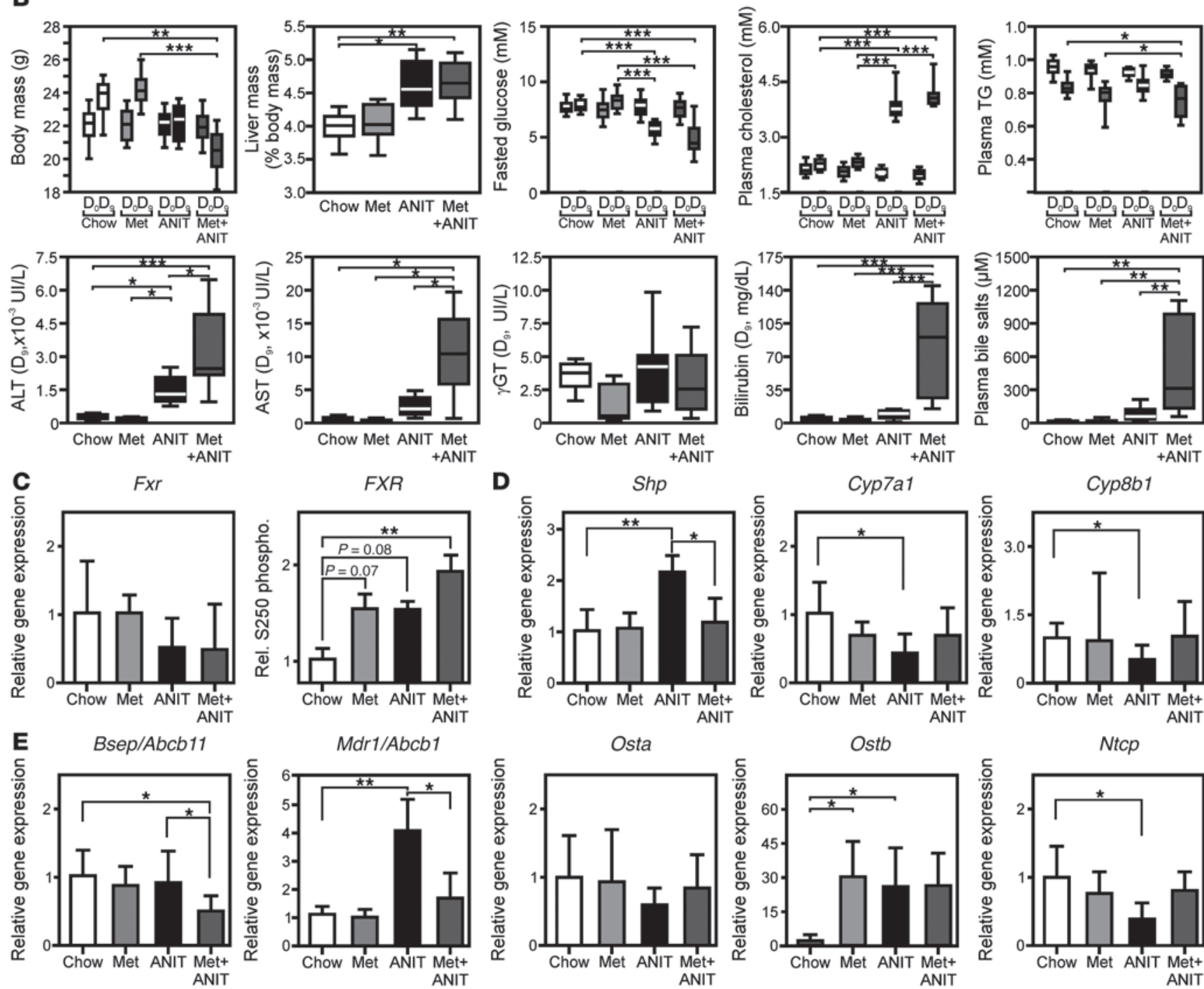

D
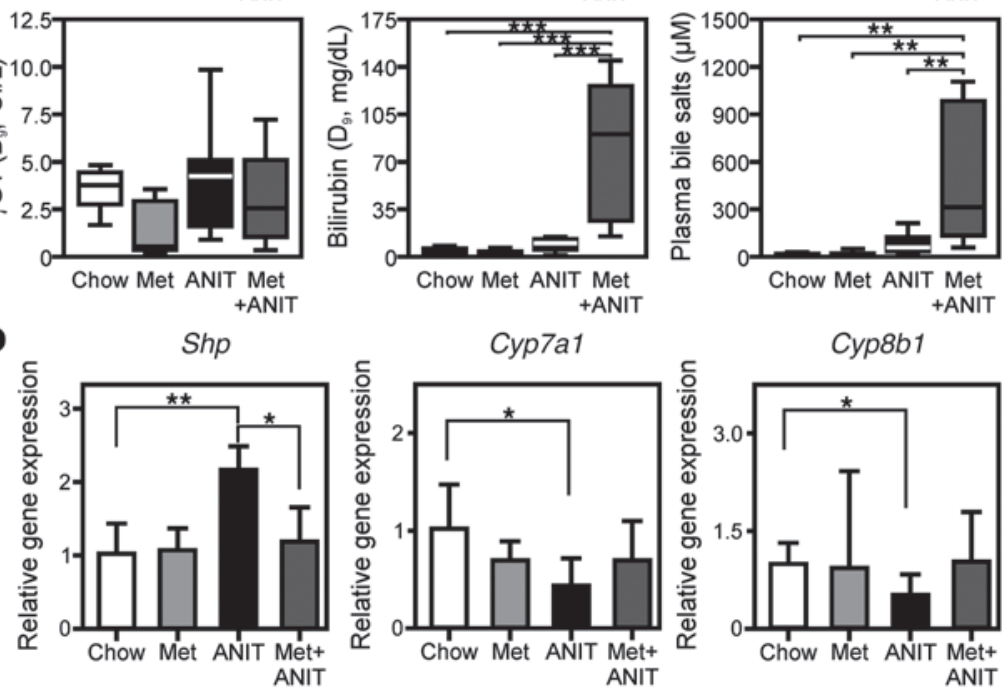

Cyp7a1

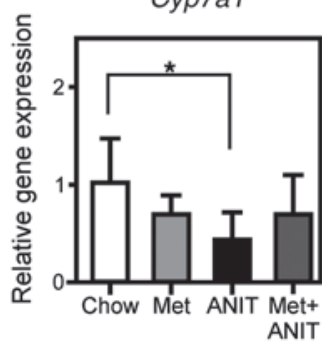

Ostb
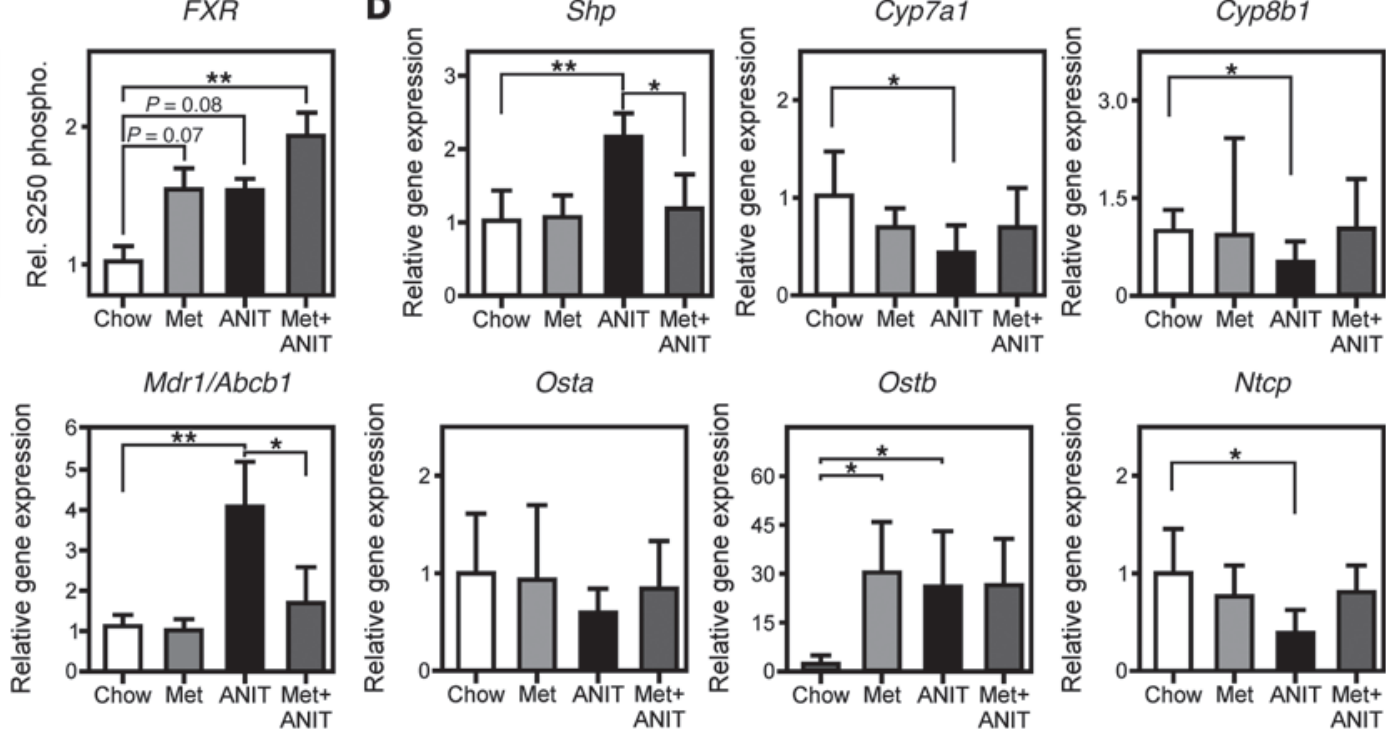

Figure 10

Metformin aggravates intrahepatic cholestasis. C57BL/6 mice were treated or not with metformin for 7 days, then gavaged with ANIT. (A) Liver histology in ANIT-induced cholestasis. H\&E-stained liver sections from each treatment group are shown. Right panel; quantification of lesion area in each treatment group. Results are expressed as lesion area normalized to slice area $(n=6-8)$. Original magnification, $\times 20$. (B) Biometric and plasma biochemical parameters in control (chow), metformin-, ANIT-, or ANIT and metformin-treated mice. $\mathrm{D}_{0}$ : day 0; $\mathrm{D}_{9}$ : day 9 (end of treatment). (C) FXR expression and phosphorylation levels. Total RNA or whole-cell extracts were used to assess Fxr mRNA expression by RT-qPCR or the phospho-FXR levels by IP/Western-blotting respectively. Results are expressed relative to control conditions (chow) set to 1. (D and E) FXR target gene expression in liver. mRNA levels were quantified by RT-qPCR, and results are expressed relative to a control sample (chow diet) arbitrarily set to $1 .{ }^{*} P<0.05,{ }^{* *} P<0.01 ;{ }^{* * *} P<0.005$. 
and $\operatorname{Bacs}(P<0.01)$ mRNA steady-state levels in the liver and significantly repressed the expression of the indirect FXR target genes Ntcp, Cyp7a1, and Cyp8b1 $(P<0.05)$. Metformin alone did not alter the basal expression of these FXR target genes (nor upon long term treatment in high fat-fed mice and obese diabetic patients; Supplemental Figure 14). Most strikingly, metformin blunted the TCA-induced expression of hepatic Shp, Bsep, Mrp2, Bacs, and Baat (Figure 8A). Thus, in vivo metformin treatment selectively affects FXR-mediated upregulation of liver genes involved in BA conjugation, uptake, and export.

$A M P K$ activation affects FXR-regulated BA enterohepatic cycling. BA overload, while not inducing symptoms of global toxicity that could translate into weight loss or increased liver mass (Figure 8B and Supplemental Figure 15), decreased plasma glucose, total cholesterol, and triglycerides (Figure 8B) as expected from FXR activation (23). Increases in plasma ALT and AST were also observed, illustrating the cholestatic effect of the TCA load. Plasma bile salt levels remained unchanged compared with those of control mice, indicative of the successful adaptive response to BA loading, illustrated by an increased bile flow and increased fecal BA excretion (Figure 8C), in line with the increased expression of hepatic canalicular BA transporters Bsep and Mrp2 and previous reports $(24,25)$. Metformin alone did not modify any of the measured parameters, which is illustrated by its absence of effect on gene expression in liver (Figure 8A). In contrast, concomitant treatment with TCA and metformin led to a strong increase of plasma BA levels and decreased fecal BA excretion, suggesting a negative interference with BA enterohepatic recycling similar to that observed in FXR-null mice (24). Finally, Western blot analysis of liver extracts confirmed the increased phosphorylation of FXR at S250 upon concomitant treatment by metformin and TCA (Figure 8D and Supplemental Figure 16). To assess the contribution of FXR to the observed metformin effect, liver-specific FXR KO mice (LFxr-l-; ref. 26) or WT littermates $\left(\mathrm{LFrr}^{+/+}\right)$were studied (Supplemental Figure 17). Mice were fed either a chow diet supplemented or not with $0.5 \%$ TCA with or without metformin (50-80 mpk/day) for 5 days, and gene expression in liver and ileum was monitored. As expected, FXR target genes were not regulated in the liver of $\mathrm{LFxr} /$-mice in response to TCA treatment (Figure 9A), but were induced in the ileum (Figure 9B). Metformin fully inhibited the TCA-induced, FXRdependent induction of hepatic (Figure 9A) and ileal (Figure 9B) FXR target genes, in agreement with previous in vitro, ex vivo, and in vivo data. Metformin did not influence basal gene expression in the liver of LFxr ${ }^{-1}$ mice (Figure 9A), indicating that metformin requires FXR to regulate the expression of these target genes. The TCA load mimics an increase in BA pool size, with which WT mice cope by increasing the fecal BA excretion rate (Figure 9C; see also Figure 8C). The hepatic canalicular secretion defect generated by the liver-specific Fxr deletion strongly affected fecal BA excretion. Whereas metformin inhibited the fecal BA excretion in mice fed with TCA, this was not observed in $\mathrm{LFxr}^{-/}$mice, demonstrating the essential role of liver FXR in response to metformin.

Metformin aggravates liver injury in a model of intrahepatic cholestasis. Characterized by an interruption of bile flow and subsequent accumulation of BA in the liver, increased plasma BA, and decreased $\mathrm{BA}$ excretion, cholestasis is a rare but serious condition in metformin-treated patients (27). As a population of metformin-treated diabetic patients showed a mild but significant trend to exhibiting increased plasma $\gamma$-GT levels (Supplemental Figure 18), a biochemical marker of liver damage associated with obstructive cholesta- sis in humans, and since TCA/metformin-treated mice exhibited increased plasma BA concentration and decreased BA excretion (Figure 8), the impact of metformin treatment in a cholestatic condition was assessed. The $\alpha$-naphthyl isothiocyanate (ANIT) model mimics intrahepatic cholestasis, in which FXR activation has been shown to be protective against liver damage $(9,10)$. The effect of metformin in the ANIT model was assessed by treating C57BL6/J mice with metformin for 7 days prior to ANIT administration. Forty-eight hours after ANIT treatment, the extent of liver damage was investigated by histopathological analysis of H\&E-stained liver sections (Figure 10A). While control and metformin-treated mice showed normal liver histology, ANIT-treated mice showed necrotic lesions that were more pronounced upon metformin treatment. Macroscopic damage to the liver by ANIT was also illustrated by increased liver mass. Biochemical markers of liver injury (ALT, AST, bilirubin) were elevated upon ANIT treatment and further increased upon metformin treatment (Figure 10B). Similarly, ANIT-induced plasma BA concentrations further increased upon metformin treatment, consistent with the cumulative effect of TCA and metformin on this parameter (Figure 10B).

Adaptive changes in transporter gene expression in cholestasis have been shown to be FXR dependent (28), suggesting that FXR is activated in this model. In this setting, neither FXR mRNA (Figure 10C) nor protein (Supplemental Figure 19) expression varied significantly. However, FXR S250 phosphorylation was significantly increased upon ANIT/metformin treatment $(P<0.001)$ and showed a similar trend, although it was nonsignificant upon metformin treatment. Interestingly, ANIT treatment itself caused a moderate increase in FXR phosphorylation (Figure 10C and Supplemental Figure 19), in line with the reported mitochondrial dysfunction and AMPK activation in cholestasis $(29,30)$. The expression of direct or indirect FXR target genes involved in BA synthesis (Shp, Cyp7a1, Cyp8b1) or transport (Bsep, Mdr1, Osta/b, Ntcp) was therefore assessed in our model (Figure 10, D and E). ANIT treatment significantly induced the expression of the direct FXR target genes $\operatorname{Sh} p(P<0.01), M d r 1$ $(P<0.01)$, and $O s t b(P<0.05)$, while leaving unchanged that of Bsep and Osta. The expression of the indirect FXR target genes Ntcp, Cyp7a1, and Cyp8b1 $(P<0.05)$ was repressed, in agreement with increased FXR activity. While metformin only affected the basal expression of Ostb, it blunted ANIT's effect for each measured gene. While ANIT did not induce Bsep expression in our model, it decreased Bsep mRNA in the presence of metformin. This response is similar to that observed in ANIT-treated Fxr-KO mice (10), suggesting again that metformin impairs FXR activity. Taken together, our data indicate that metformin treatment sensitizes mice to liver damage caused by intrahepatic cholestasis by negatively interfering with FXR-dependent gene expression.

\section{Discussion}

This study has identified a pathway via which the energy-sensitive protein kinase AMPK regulates the nuclear BA sensor FXR. Using a biochemical approach, we have shown that AMPK subunits bind directly to FXR and that the AMPK complex acts as a FXR repressor through direct phosphorylation. The phosphorylation of the FXR serine residue 250 by AMPK and the inhibition of the liganddependent activation of FXR by AMPK suggested a functional dependency of the AF2 domain on the hinge region. As a result, coactivator loading on chromatin-bound FXR was prevented. Although available crystallographic structures of FXR rule out a direct steric interference of the phosphorylated hinge region with 
the FXR/LXXLL coactivator interface, the hinge region is considered as a domain exerting allosteric action whose structure is critical for ligand-dependent NR-mediated transactivation, as confirmed by recent crystallographic studies (31). Accordingly, phosphorylation of the hinge domain of several NRs has been reported, with varying consequences on the transcriptional activity of these transcription factors and distinct mechanisms of action (32). Only a few reports, however, point to a link between the AMPK signaling cascade and the NR signaling pathways. Correlative evidence shows that dysregulation of ROR $\alpha$ signaling in mouse skeletal muscle perturbs AMPK activity (33). A direct posttranslational modification of HNF $4 \alpha$ by AMPK inhibits its ability to homodimerize and reduces HNF $4 \alpha$ polypeptide stability (34). While these data suggest that AMPK activation may control the transcriptional activity of a broader set of NRs, our Gal4-based assay shows that AMPK acts directly on FXR, but not on RXR $\alpha, \operatorname{LXR} \alpha$, or LXR $\beta$. The AMPK activator metformin accumulates preferentially in the small intestine and the liver (35), and 25 NRs display a noticeable expression in mouse liver and/or intestine (36). They contain consensus AMPK phosphorylation sites, rendering them potentially sensitive to metformin-activated AMPK. However, the 25 amino

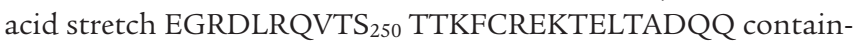
ing the unique FXR AMPK phosphorylation site is not conserved in the NR superfamily, implying that the role of AMPK in regulating NR activity has to be examined on a case-by-case basis.

Recently, SRC2 was shown to be phosphorylated and activated by AMPK, thereby selectively increasing Bsep expression in a FXRdependent manner. Conversely, the double AMPK $\alpha 1 / \alpha 2 \mathrm{KO}$ blunted Bsep expression and affected hepatic BA content (15). This contrasts with the lack of effect of liver LKB1 KO, which strongly downregulates AMPK $\alpha 1$ and $\alpha 2$ activity, yet has no effect on Bsep expression (37). Our findings demonstrate that AMPK activation exerts an opposite effect on the transcriptional activity of ligandactivated FXR, while a trend, although statistically not significant, to increase the basal expression of Bsep/BSEP was observed upon pharmacological AMPK activation in vivo (Figure 8A and Supplemental Figure 14, A and B) and in vitro (Figure 3B). Our ChIP-PCR and ChIP-seq data show that RXR-FXR heterodimer assembly to the BSEP promoter is strongly favored by FXR agonism in HepG2 cells (Supplemental Figure 20), thus providing a mechanistic basis for the very moderate effect of AMPK-dependent activation of this FXRE-driven gene in our in vitro cellular model. Therefore, the observed AMPK-mediated repression and its pathological consequences relate thus only to conditions of increased hepatic BA content, i.e., (pre)cholestatic conditions.

FXR displays a clear tissue-specific expression pattern, with a high level of expression in liver as well as in the small intestine. In these organs, it plays a critical role in the regulation of the BA enterohepatic cycle, notably through the control of the expression of intestinal bile salt transporters and of BA biosynthetic enzymes. In vivo activation of AMPK could thus be predicted to severely impinge on BA homeostasis. Indeed, metformin administration perturbed BA fecal elimination upon BA loading, in line with its ability to block the adaptive increased expression of the hepatic canalicular exporters Bsep and Mrp2. Similarly, metformin increased liver injury in a model of intrahepatic cholestasis, which can be attributed at least in part to its ability to prevent increased Shp expression, whose knockout sensitizes mice to obstructive cholestasis (38). Surprisingly, selective FXR target genes are inhibited upon AMPK activation in vivo, while AMPK activation has a gen- eral repressive effect on FXR-mediated transcription in hepatocytic and enterocytic cell lines in vitro. While the mechanistic basis of this selective sensitivity deserves further investigation, this suggests that the underlying transcriptional activation mechanisms by FXR are gene and tissue specific and/or may reflect distinct FXR-controlled processes to alleviate chromatin-mediated transcriptional repression. It is worth noting that predicting in vivo transcriptional outcomes from in vitro experiments may be difficult. Indeed, the expression of the lysine methyl-transferase MLL3, a component of the coactivator complex ASCOM required for efficient FXR-dependent activation of BSEP and MRP2, was found to be decreased in the liver of bile duct-ligated mice (39). As FXR binding to chromatin is not affected by metformin treatment in vitro or in vivo, it is likely that pathophysiological conditions may strongly affect the composition of transcriptional complexes tethered to DNA-bound receptor heterodimers, as recently shown for PPAR $\gamma$ (40).

Metformin is a well-tolerated and efficient antidiabetic drug with relatively few side effects, the most common being gastrointestinal disturbances, and much more rarely, lactic acidosis. For this reason, metformin is contraindicated in patients with liver disease. However, a few cases of reversible, metformin-induced hepatic cholestasis have been reported (41-44), underlining the risk of idiosyncratic hepatotoxicity (27) for this increasingly prescribed drug in metabolic diseases, which is also currently being evaluated in cancer chemotherapy. Our results also point to the need for evaluating AMPK-targeting compounds, which are potential efficient drugs in treating T2D (45), for liver toxicity and their ability to interfere with the FXR signaling pathway, whose activity substantially affects BA, glucose, and lipid metabolism (2). The recent notion that metformin may exert its beneficial metabolic effects in genetically AMPK-deficient mice (46), at least in part through inhibition of the glucagon/cAMP signaling pathway in hepatocytes (47), indeed calls for the identification of glucosenormalizing drugs acting independently of AMPK.

Many physiological signals are known to activate AMPK (48), among which energy deprivation, hence decreased glucose availability, plays a major role. Our preliminary results suggest that FXR S250 phosphorylation varies as a function of extracellular glucose concentration (Supplemental Figure 21). This adds an additional layer of physiological control of FXR activity by AMPK, whose activity has been shown to follow diurnal variations in mouse liver (49). Whether reported circadian variations of FXR target genes are controlled, at least partially, through AMPK is currently under investigation.

\section{Methods}

Materials, cell lines, and plasmids. Chemicals, cell lines, DNA constructs, transient transfections, recombinant protein, and antibody information can be found in the Supplemental Information.

Immunofluorescence and PLA. PLA was performed as follows: $1 \times 10^{5}$ HepG2 cells per well were grown in 12 -well plates with coverslide. After 24 hour incubation with the indicated compounds ( $2 \mu \mathrm{M}$ GW4064 and/or $2 \mathrm{mM} \mathrm{AICAR}$ ), cells were washed with $1 \times$ PBS and fixed in $4 \%$ paraformaldehyde for 20 minutes at room temperature (RT). Permeabilization of cells was performed in $0.1 \%$ Triton X-100 for 10 minutes at $37^{\circ} \mathrm{C}$. The PLA assay was carried out according to manufacturer's instructions (Duolink; Olink Biosciences) using anti-FXR (PP-A9033A-00) and antiAMPKa (40H9, \#2535) antibodies. Slides were mounted in fluorescence mounting medium (Invitrogen) with DAPI for nuclear staining. Images were acquired using a Leica DMI6000B microscope. 
Immunofluorescence studies were carried out by fixing and permeabilizing cells as above. Cells were incubated with primary antibody overnight at $4^{\circ} \mathrm{C}$ in a humid chamber After washing in $1 \times \mathrm{PBS}$, cells were incubated with the appropriated fluorescent secondary antibody coupled to Alexa Fluor 488 for 30 minutes at RT. Images were acquired as described above.

RNA extraction, $c D N A$ synthesis, and real-time RT-PCR. Total RNA was extracted using RNEasy (QIAGEN) according to the manufacturer's instructions. RT-qPCR methodology information can be found in the Supplemental Information.

RNA interference experiments. $1.5 \times 10^{5}$ HepG2 cells were seeded in 6-well clusters and transfected the next day with a mix of $10 \mathrm{nM}$ siRNA and INTERFERin (Polyplus Transfection). Cells were left for 36 hours and treated as indicated in the text. siRNAs (Thermo Fisher Scientific-Dharmacon) were ON-TARGETplus nontargeting pool and NR1H4, STK11, PRKAA1, and PRKAA2 ON-TARGETplus SMARTpools.

GST pull-down assays. Glutathione S-transferase (GST) fusion protein expression and purification and GST pull-down assays were performed as previously described (50) with modifications. Detailed procedures can be found in the Supplemental Methods.

ChIP assays. ChIP assays were performed as described previously (51). Detailed information can be found in the Supplemental Methods.

In vitro phosphorylation assay. Purified FXR (15 pmol) was incubated for 60 minutes at RT with purified AMPK ( $\alpha 2: \beta 2: \gamma 1,500 \mathrm{ng})$ (Floralis) in AMPK buffer (250 mM HEPES, pH 7.4, $62.5 \mathrm{mM} \mathrm{MgCl}_{2}, 125 \mathrm{mM}$ $\beta$-glycerophosphate, $1 \mathrm{mM}$ DTT, $50 \mu \mathrm{M}$ AMP). ATP was used at $2 \mathrm{mM}$ with $5 \mu \mathrm{Ci}\left[{ }^{32} \mathrm{P}\right]-\gamma$ ATP $(10 \mathrm{mCi} / \mathrm{ml})$.

Mass spectrometric analyses. For mass fingerprinting, protein samples resolved by SDS-PAGE were detected by colloidal blue staining. Bands were digested with trypsin and analyzed by Maldi-TOF mass spectrometry. The SwissProt database was interrogated through the MS-Digest Search program. The complete protocol has been detailed elsewhere (52).

A detailed procedure for the phosphorylation site determination can be found in the Supplemental Methods.

In vivo experiments. C57BL6/J mice were purchased from Charles River Laboratories and housed in a temperature-controlled room $\left(22-24^{\circ} \mathrm{C}\right)$ with a relative humidity of $36 \%-80 \%$, and 12 -hour light $/ 12$-hour dark cycles. Mice were fed ad libitum, with free access to water. Mice $(n=8$ / group) were randomized by body weight, serum glucose, cholesterol, and triglyceride levels and received either (a) a standard rodent chow diet (A03-10 UAR, control), (b) a standard rodent chow diet and metformin $(0.5 \mathrm{~g} / \mathrm{l}$ in drinking water, ca $50-80 \mathrm{mpk} / \mathrm{d})$, (c) a standard rodent chow diet containing $0.5 \%$ (wt/wt) TCA, or (d) a standard rodent chow diet containing $0.5 \%$ (wt $/ \mathrm{wt}$ ) TCA and metformin for 5 days. Mice were sacrificed by cervical dislocation after 6 hours of fasting, and tissues were removed, weighed and immediately frozen in liquid $\mathrm{N}_{2}$. A similar experiment was performed in which TCA was substituted for GW4064 (30 mpk/d by gavage in $1 \%$ CMC, $1 \%$ Tween 80$)$ and metformin by AICAR $(200 \mathrm{mpk} / \mathrm{d}$ by intraperitoneal injection).

Chemically induced intrahepatic cholestasis. After 7 days with free access to water or water supplemented with metformin $(0.5 \mathrm{~g} / \mathrm{l})$ and chow diet, C57BL6/J male mice ( $n=8$ per group) were gavaged with either vehicle (olive oil) or ANIT at $75 \mathrm{mpk}$. Food and water intake, biometric parameters, plasma glucose, total cholesterol, HDL cholesterol, BAs and triglyceride concentrations were measured 48 hours later. Detailed information about biometric and biochemical parameter, BA assays, and liver histology can be found information the Supplemental Methods.

Statistics. Raw data were processed using Prism v5.0 (GraphPad). Results are expressed as mean $\pm \operatorname{SEM}(n=3-5$ for in vitro experiments, $n=8$ for in vivo experiments), and groups were compared using either an unpaired $t$ test or 1-way ANOVA followed by Tukey's post-hoc test. $P$ values of less than 0.05 were considered significant. Box-and-whiskers plots show the median, with 25 th to 75 th percentile intervals.

Study approval. Experimental protocols were approved by the Lille Pasteur Institute ethical committee and carried out in agreement with European Union (EEC no. 07430) and French ethical guidelines. The ABOS study is registered at ClinicalTrials.gov under the identifier NCT01129297.

\section{Acknowledgments}

F. Lien was supported by a fellowship from the French Ministry for Education and Research and from the Société Française d'Endocrinologie (SFE). This work was supported by grants from INSERM, and EU grants Hepadip (\#018734), Agence Nationale de la Recherche (ANR) (FXRen), EGID (ANR-10-LABX-46), Région Nord-Pas de Calais, Fond Européen de Développement Régional (FEDER), and Cost Action BM0602. We wish to thank D. Dombrowicz and S. Fleury for help with initial in vivo experiments, J. Eeckhoute for help with ChIP-seq data analysis, C. Brand and V. Lardeux for technical support, A. Tailleux and E. Vallée for BA assays, and P. Mathurin for helpful discussions. B. Staels is a member of the Institut Universitaire de France.

Received for publication August 30, 2013, and accepted in revised form December 5, 2013.

Address correspondence to: Philippe Lefebvre or Bart Staels, INSERM U1011-Bâtiment J\&K; Faculté de Médecine de Lille-Pôle Recherche, Boulevard du Pr Leclerc, 59045 Lille cedex, France. Phone: 33.3.20974220; Fax: 33.3.20974201; E-mail: philippeclaude.lefebvre@inserm.fr. (P. Lefebvre). Phone: 33.3.877825; Fax: 33.3.20974201; E-mail: bart.staels@pasteur-lille.fr(B. Staels).
1. Nguyen A, Bouscarel B. Bile acids and signal transduction: role in glucose homeostasis. Cell Signal. 2008;20(12):2180-2197.

2. Lefebvre P, Cariou B, Lien F, Kuipers F, Staels B. Role of bile acids and bile acid receptors in metabolic regulation. Physiol Rev. 2009;89(1):147-191.

3. Zhang Y, Castellani LW, Sinal CJ, Gonzalez FJ, Edwards PA. Peroxisome proliferator-activated receptor-gamma coactivator 1alpha (PGC-1alpha) regulates triglyceride metabolism by activation of the nuclear receptor FXR. Genes Dev. 2004; 18(2):157-169.

4. Ananthanarayanan M, Li S, Balasubramaniyan N, Suchy FJ, Walsh MJ. Ligand-dependent activation of the farnesoid X-receptor directs arginine methylation of histone H3 by CARM1. J Biol Chem. 2004; 279(52):54348-54357.

5. Rizzo G, Renga B, Antonelli E, Passeri D, Pellic- ciari R, Fiorucci S. The methyl transferase PRMT1 functions as co-activator of farnesoid $\mathrm{X}$ receptor (FXR)/9-cis retinoid X receptor and regulates transcription of FXR responsive genes. Mol Pharmacol. 2005;68(2):551-558.

6. Pineda Torra I, Freedman LP, Garabedian MJ. Identification of DRIP205 as a coactivator for the Farnesoid X receptor. J Biol Chem. 2004; 279(35):36184-36191.

7. Kemper JK. Regulation of FXR transcriptional activity in health and disease: emerging roles of FXR cofactors and post-translational modifications. Biochim Biophys Acta. 2011;1812(8):842-850.

8. Inagaki T, et al. Fibroblast growth factor 15 functions as an enterohepatic signal to regulate bile acid homeostasis. Cell Metab. 2005;2(4):217-225.

9. Liu Y, et al. Hepatoprotection by the farnesoid X receptor agonist GW4064 in rat models of intra- and extrahepatic cholestasis. J Clin Invest. 2003; 112(11):1678-1687.

10. Cui YJ, Aleksunes LM, Tanaka Y, Goedken MJ, Klaassen CD. Compensatory induction of liver efflux transporters in response to ANIT-induced liver injury is impaired in FXR-null mice. Toxicol Sci. 2009;110(1):47-60.

11. Fiorucci S, et al. Protective effects of 6-ethyl chenodeoxycholic acid, a farnesoid $\mathrm{X}$ receptor ligand, in estrogen-induced cholestasis. J Pharmacol Exp Ther. 2005;313(2):604-612.

12. Canto C, et al. AMPK regulates energy expenditure by modulating NAD + metabolism and SIRT1 activity. Nature. 2009;458(7241):1056-1060.

13. Fulco M, et al. Glucose restriction inhibits skeletal myoblast differentiation by activating SIRT1 through AMPK-mediated regulation of Nampt. Dev Cell. 2008;14(5):661-673. 
14. Lan F, Cacicedo JM, Ruderman N, Ido Y. SIRT1 modulation of the acetylation status, cytosolic localization, and activity of LKB1. Possible role in AMP-activated protein kinase activation. J Biol Chem. 2008;283(41):27628-27635

15. Chopra AR, et al. Cellular energy depletion resets whole-body energy by promoting coactivatormediated dietary fuel absorption. Cell Metab. 2011; 13(1):35-43

16. Duran-Sandoval D, et al. Glucose regulates the expression of the farnesoid $\mathrm{X}$ receptor in liver. Diabetes. 2004;53(4):890-898.

17. Surapureddi S, et al. Identification of a transcriptionally active peroxisome proliferator-activated receptor alpha -interacting cofactor complex in rat liver and characterization of PRIC285 as a coactivator. Proc Natl Acad Sci U S A. 2002;99(18):11836-11841.

18. Hardie DG. AMP-activated/SNF1 protein kinases: conserved guardians of cellular energy. Nat Rev Mol Cell Biol. 2007;8(10):774-785.

19. Reznick RM, Shulman GI. The role of AMP-activated protein kinase in mitochondrial biogenesis. JPhysiol. 2006;574(pt 1):33-39.

20. Frankenberg T, Rao A, Chen F, Haywood J, Shneider BL, Dawson PA. Regulation of the mouse organic solute transporter $\alpha-\beta$, Ostalpha-Ostbeta, by bile acids. Am J Physiol Gastrointest Liver Physiol. 2006; 290(5):G912-G922.

21. Kodiha M, Rassi JG, Brown CM, Stochaj U. Localization of AMP kinase is regulated by stress, cell density, and signaling through the MEK $\rightarrow$ ERK $1 / 2$ pathway. Am J Physiol Cell Pbysiol. 2007; 293(5):C1427-C1436.

22. Stein SC, Woods A, Jones NA, Davison MD, Carling $D$. The regulation of AMP-activated protein kinase by phosphorylation. Biochem J. 2000;345(pt 3):437-443.

23. Zhang Y, et al. Activation of the nuclear receptor FXR improves hyperglycemia and hyperlipidemia in diabetic mice. Proc Natl Acad Sci U S A. 2006; 103(4):1006-1011.

24. Sinal CJ, Tohkin M, Miyata M, Ward JM, Lambert G, Gonzalez FJ. Targeted disruption of the nuclear receptor FXR/BAR impairs bile acid and lipid homeostasis. Cell. 2000;102(6):731-744.

25. Zollner G, et al. Coordinated induction of bile acid detoxification and alternative elimination in mice: role of FXR-regulated organic solute transporter-alpha/beta in the adaptive response to bile acids. Am J Physiol Gastrointest Liver Physiol. 2006; 290(5):G923-G932.
26. Prawitt J, et al. Farnesoid X receptor deficiency improves glucose homeostasis in mouse models of obesity. Diabetes. 2011;60(7):1861-1871.

27. Miralles-Linares F, Puerta-Fernandez S, BernalLopez MR, Tinahones FJ, Andrade RJ, GomezHuelgas R. Metformin-induced hepatotoxicity. Diabetes Care. 2012;35(3):e21.

28. Stedman C, et al. Benefit of farnesoid X receptor inhibition in obstructive cholestasis. Proc Natl Acad Sci U S A. 2006;103(30):11323-11328.

29. Schulz S, et al. Progressive stages of mitochondrial destruction caused by cell toxic bile salts. Biochim Biophys Acta. 2013;1828(9):2121-2133.

30. Hsu CC, et al. Mitochondrial dysfunction represses HIF-1alpha protein synthesis through AMPK activation in human hepatoma HepG2 cells. Biochim Biophys Acta. 2013;1830(10):4743-4751.

31. Orlov I, Rochel N, Moras D, Klaholz BP. Structure of the full human RXR/VDR nuclear receptor heterodimer complex with its DR3 target DNA. EMBOJ. 2012;31(2):291-300.

32. Berrabah W, Aumercier P, Lefebvre P, Staels B. Control of nuclear receptor activities in metabolism by post-translational modifications. FEBS Lett. 2011; 585(11):1640-1650

33. Raichur S, et al. Identification and validation of the pathways and functions regulated by the orphan nuclear receptor, ROR $\alpha 1$, in skeletal muscle. Nucleic Acids Res. 2010;38(13):4296-4312.

34. Hong YH, Varanasi US, Yang W, Leff T. AMPactivated protein kinase regulates $\mathrm{HNF} 4 \alpha$ transcriptional activity by inhibiting dimer formation and decreasing protein stability. J Biol Chem. 2003; 278(30):27495-27501.

35. Wilcock C, Bailey CJ. Accumulation of metformin by tissues of the normal and diabetic mouse. Xenobiotica. 1994;24(1):49-57.

36. Bookout AL, Jeong Y, Downes M, Yu RT, Evans RM, Mangelsdorf DJ. Anatomical profiling of nuclear receptor expression reveals a hierarchical transcriptional network. Cell. 2006;126(4):789-799.

37. Woods A, et al. LKB1 is required for hepatic bile acid transport and canalicular membrane integrity in mice. Biochem J. 2011;434(1):49-60.

38. Park YJ, et al. Loss of orphan receptor small heterodimer partner sensitizes mice to liver injury from obstructive cholestasis. Hepatology. 2008; 47(5):1578-1586

39. Ananthanarayanan M, et al. Histone $\mathrm{H} 3 \mathrm{~K} 4$ trimethylation by MLL3 as part of ASCOM complex is criti- cal for NR activation of bile acid transporter genes and is downregulated in cholestasis. Am J Physiol Gastrointest Liver Physiol. 2011;300(5):G771-G781.

40. Lefebvre B, et al. Proteasomal degradation of retinoid $X$ receptor $\alpha$ reprograms transcriptional activity of PPAR $\gamma$ in obese mice and humans. J Clin Invest. 2010;120(5):1454-1468.

41. Desilets DJ, Shorr AF, Moran KA, Holtzmuller KC. Cholestatic jaundice associated with the use of metformin. Am J Gastroenterol. 2001;96(7):2257-2258.

42. Kutoh E. Possible metformin-induced hepatotoxicity. Am J Geriatr Pharmacother. 2005;3(4):270-273.

43. Nammour FE, Fayad NF, Peikin SR. Metformininduced cholestatic hepatitis. Endocr Pract. 2003; 9(4):307-309.

44. Biyyani RS, Battula S, Erhardt CA, Korkor K. Metformin-induced cholangiohepatitis. BMJ Case Rep. 2009;2009:bcr09.2008.0950.

45. Zhang BB, Zhou G, Li C. AMPK: an emerging drug target for diabetes and the metabolic syndrome. Cell Metab. 2009;9(5):407-416.

46. Foretz M, et al. Metformin inhibits hepatic gluconeogenesis in mice independently of the LKB1/ AMPK pathway via a decrease in hepatic energy state. J Clin Invest. 2010;120(7):2355-2369.

47. Miller RA, Chu Q, Xie J, Foretz M, Viollet B, Birnbaum MJ. Biguanides suppress hepatic glucagon signalling by decreasing production of cyclic AMP. Nature. 2013;494(7436):256-260.

48. Hardie DG, Ross FA, Hawley SA. AMPK: a nutrient and energy sensor that maintains energy homeostasis. Nat Rev Mol Cell Biol. 2012;13(4):251-262.

49. Lamia KA, et al. AMPK regulates the circadian clock by cryptochrome phosphorylation and degradation. Science. 2009;326(5951):437-440.

50. Rachez C, et al. A novel protein complex that interacts with the vitamin D- 3 receptor in a liganddependent manner and enhances VDR transactivation in a cell-free system. Genes Dev. 1998; 12(12):1787-1800.

51. Flajollet S, Lefebvre B, Rachez C, Lefebvre P. Distinct roles of the steroid receptor coactivator 1 and of MED1 in retinoid-induced transcription and cellular differentiation. J Biol Chem. 2006; 281(29):20338-20348.

52. Sacchetti P, Carpentier R, Segard P, Olive-Cren $\mathrm{C}$, Lefebvre $\mathrm{P}$. Multiple signaling pathways regulate the transcriptional activity of the orphan nuclear receptor NURR1. Nucleic Acids Res. 2006; 34(19):5515-5527. 